\title{
TWO LIMIT CYCLES IN LIÉNARD PIECEWISE LINEAR DIFFERENTIAL SYSTEMS
}

\author{
JAUME LLIBRE ${ }^{1}$, ENRIQUE PONCE $^{2}$ AND CLÀUDIA VALLS ${ }^{3}$
}

\begin{abstract}
Some techniques for studying the existence of limit cycles for smooth differential systems are extended to continuous piecewise-linear differential systems. Rigorous new results are provided on the existence of two limit cycles surrounding the equilibrium point at the origin for systems with three zones separated by two parallel straight lines without symmetry.
\end{abstract}

\section{Introduction AND STATEMENT OF THE MAin RESUlts}

One of the most interesting problems in the qualitative theory of planar polynomial differential systems is the study of their limit cycles, known as the famous second part of the 16-th Hilbert problem [13]. Due to the fact that this Hilbert problem becomes up to now intractable (see $[16,18]$ ), Smale in [30] proposed to study this problem restricting it to polynomial Liénard differential systems. In the case of smooth Liénard systems there are many results on the non-existence, existence and uniqueness of limit cycles, see for instance $[1,4,6,12,17,20,25,31,33]$. Going beyond the smooth case a natural step is to allow non-smoothness while keeping the continuity, as it has been done in some previous works [9, 14, 15, 21, 24].

While the majority of results for piecewise linear differential systems deal with two zones separated by one straight line or three zones separated by two parallel straight lines with symmetry and study the existence of at most one limit cycle, in this paper we go beyond and focus the attention to non-symmetric systems. Particular cases of such non-symmetric systems but assuming a certain symmetry for the sign of their determinants and traces in the three regions and allowing only one equilibrium point have been studied in $[28,3]$. The quoted authors are able to show the existence of two limit cycles surrounding the only equilibrium under adequate hypotheses. Similar results have been recently obtained in [19] by considering perturbations of systems without sign-symmetric traces but under rather non-generic hypotheses. In all the quoted cases, the location of the equilibrium is out of the central zone for having two limit cycles.

Here, we do not assume any symmetry at all and give an extensive list of cases were we prove the existence of two limit cycles surrounding the origin, a new result in this field. In [26] exploiting the fact that there are situations where, by moving only the parameter given by the trace of the central zone, it is possible to pass from a system with two zones to a system with three zones, the authors were able to prove the existence of at least two limit cycles surrounding the equilibrium at the origin in some particular cases. The characterization of all possible cases with two

2010 Mathematics Subject Classification. Primary 34C05. Secondary 34C23.

Key words and phrases. Nonlinear control systems, periodic orbits, limit cycles, Liénard piecewise linear differential systems. 
limit cycles is far from being completely solved and this paper is the first rigorous paper towards this goal exploiting all possibilities in which we are able to prove the existence of at least two limit cycles surrounding the equilibrium at the origin. This is the aim of this paper and the techniques used to achieve this goal are not the same as the ones in [26].

More precisely, in this work we will study the limit cycles of the Liénard piecewise linear differential systems

$$
\frac{d x}{d \tau}=x^{\prime}=F(x)-y, \quad \frac{d y}{d \tau}=y^{\prime}=g(x),
$$

where

and

$$
F(x)= \begin{cases}T_{L}(x+u)-T_{C} u & \text { if } x \leq-u \\ T_{C} x & \text { if }-u \leq x \leq v, \\ T_{R}(x-v)+T_{C} v & \text { if } x \geq v\end{cases}
$$

$$
g(x)= \begin{cases}L(x+u)-u & \text { if } x \leq-u \\ x & \text { if }-u \leq x \leq v, \\ R(x-v)+v & \text { if } x \geq v\end{cases}
$$

with the constants $u$ and $v$ being positive, so that the straight lines $x=-u$ and $x=v$ split the phase plane in three regions. In the case that these systems are symmetric with respect the origin of coordinates, i.e.

$$
u=v, \quad T_{L}=T_{R}, \quad L=R .
$$

the study of their limit cycles is done, see $[2,7,9,10,23]$, and for a complete analysis the book [24].

First, we classify the equilibria of system (1).

Proposition 1. The following statements hold for the Liénard piecewise linear differential system (1).

(a) If $L \geq 0$ and $R \geq 0$, then the origin is the unique equilibrium.

(b) If $L \geq 0$ and $R<0$, then there are two equilibria, the origin and $e_{R}=$ $\left(\bar{x}_{R}, \bar{y}_{R}\right)=\left((R-1) v / R,\left(T_{C} R-T_{R}\right) v / R\right)$, which is a saddle.

(c) If $L<0$ and $R \geq 0$, then there are two equilibria: the origin and $e_{L}=$ $\left(\bar{x}_{L}, \bar{y}_{L}\right)=\left((1-L) u / L,\left(T_{L}-T_{C} L\right) u / L\right)$, which is a saddle.

(d) If $L<0$ and $R<0$, then there are three equilibria: the origin, $e_{L}$ and $e_{R}$, being $e_{L}$ and $e_{R}$ saddles.

Proof. It follows easily by direct computations because when they exist belong to the interior of each one of the three zones where the differential system is linear.

We note that the equilibrium point $e_{R}$ exists if and only if $R<0$, and when $R>0$ we say that $e_{R}$ is a virtual equilibrium. Similarly, the equilibrium point $e_{L}$ exists if and only if $L<0$, and when $L>0$ we say that $e_{L}$ is a virtual equilibrium. It follows from Proposition 1 that when the Liénard piecewise linear differential system (1) has a dynamics of focus or node type in an external zone then the corresponding focus or node is a virtual equilibrium point; however, when such dynamics is of saddle type then the saddle is always a real equilibrium point.

We now introduce some notation. When $T_{L}^{2}>4 L$ we can define $w_{L}>0$ such that $4 w_{L}^{2}=T_{L}^{2}-4 L$ and we are dealing with a dynamics of node or saddle type. 
If we also introduce $\sigma_{L}$ such that $2 \sigma_{L}=T_{L}$ then the eigenvalues are $\sigma_{L} \pm w_{L}$, with eigenvectors $\left(1, \sigma_{L} \mp w_{L}\right)^{T}$ and $L=\sigma_{L}^{2}-w_{L}^{2}$. Thus, the corresponding (real or virtual) equilibrium point has two linear invariant manifolds that intersect the straight line $x=-u$ in two points of coordinates $\left(-u, P_{L}^{ \pm}\right)$. For the focus case, that is $T_{L}^{2}<4 L$, we define $\sigma_{L}$ as before but we take $\omega_{L}>0$ such that $4 \omega_{L}^{2}=4 L-T_{L}^{2}$, so that the eigenvalues are now $\sigma_{L} \pm i \omega_{L}$.

Similarly, when $T_{R}^{2}>4 R$ we can define $w_{R}>0$ such that $4 w_{R}^{2}=T_{R}^{2}-4 R$ for the node or saddle cases. If we take $\sigma_{R}$ such that $2 \sigma_{R}=T_{R}$ then the eigenvalues are $\sigma_{R} \pm w_{R}$, with eigenvectors $\left(1, \sigma_{R} \mp w_{R}\right)^{T}$ and $R=\sigma_{R}^{2}-w_{R}^{2}$. Thus, the corresponding (real or virtual) equilibrium point has two linear invariant manifolds that intersect the straight line $x=v$ in two points of coordinates $\left(v, P_{R}^{ \pm}\right)$. For the focus case, that is $T_{R}^{2}<4 R$, we define $\sigma_{R}$ as before but we take $\omega_{R}>0$ such that $4 \omega_{R}^{2}=4 R-T_{R}^{2}$, so that the eigenvalues are now $\sigma_{R} \pm i \omega_{R}$.

We remark that the non-generic cases $L=0, R=0$, corresponding to bifurcations of equilibrium points at infinity, and the cases of improper node dynamics $T_{L}^{2}=4 L, T_{R}^{2}=4 R$, will not be included in the subsequent analysis for the sake of brevity. However, the followed approach could be extended to deal with these cases without any special difficulty.

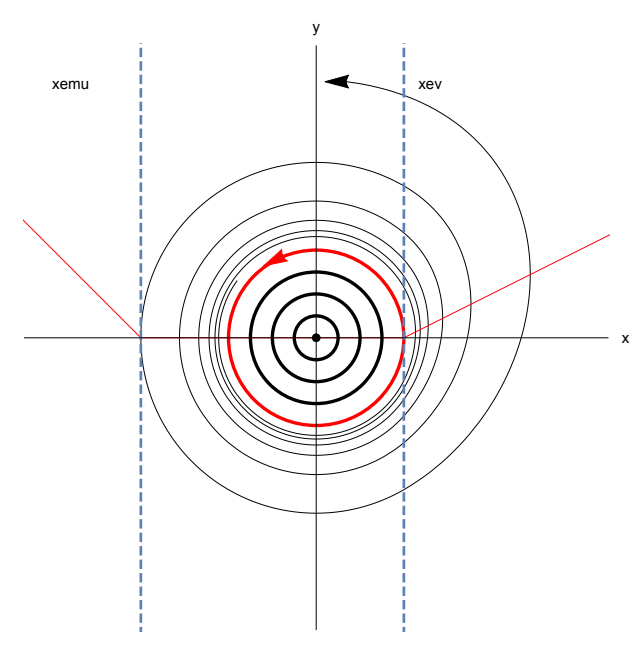

Figure 1. Phase-portrait corresponding to the case of reference $0<v<u, T_{L}<0, T_{C}=0$, and $T_{R}>0$. The outermost periodic orbit of the annulus corresponds to the circle $x^{2}+y^{2}=v^{2}$ and is unstable from outside, as $T_{R}>0$.

If we focus our attention in the cases with $T_{C}=0$, it is clear that the origin is a linear center, leading to a period annulus which is bounded by the vertical line $x=v$, being its outermost periodic orbit the one of equation $x^{2}+y^{2}=v^{2}$, see Figure 1. Furthermore, apart from the origin, the possible real or virtual equilibrium points are located at $e_{L}$ and $e_{R}$, namely

(2) $e_{L}=\left(\bar{x}_{L}, \bar{y}_{L}\right)=\left(\frac{u}{L}-u, \frac{T_{L}}{L} u\right), \quad e_{R}=\left(\bar{x}_{R}, \bar{y}_{R}\right)=\left(v-\frac{v}{R},-\frac{T_{R}}{R} v\right)$. 
Taking this situation as a main reference for our subsequent analysis, we can state the following results whose proof is given in Section 3.

Proposition 2. Consider the differential systems (1) with $0<v<u, T_{L}<0$ and $T_{C}=0$. The following statements hold.

(a) If $T_{L}^{2}>4 L$ and we take $2 \sigma_{L}=T_{L}$ and $w_{L}>0$ such that $4 w_{L}^{2}=T_{L}^{2}-4 L$, then the equilibrium point $e_{L}$ is an attractive virtual node when $L>0$ and a real saddle for $L<0$.

(a.1) When $L>0$, the invariant manifolds of the virtual node intersect the line $x=-u$ at the points $\left(-u, P_{L}^{ \pm}\right)$and such orbits enter the region $-u<x<v$ intersecting the line $x=v$ at the points $\left(v, Q_{L}^{ \pm}\right)$, where

$$
P_{L}^{ \pm}=\frac{u}{\sigma_{L} \mp w_{L}}, \quad Q_{L}^{ \pm}=-\sqrt{\left(P_{L}^{ \pm}\right)^{2}+u^{2}-v^{2}},
$$

so that $P_{L}^{-}<P_{L}^{+}<0$ and $Q_{L}^{-}<Q_{L}^{+}<0$. A first integral for the orbits above such invariant manifolds, when restricted to the region $x \leq-u$, is the function

$$
H_{L}^{N}(\tilde{x}, \tilde{y})=\log \sqrt{\left(\tilde{y}-\sigma_{L} \tilde{x}\right)^{2}-w_{L}^{2} \tilde{x}^{2}}+\frac{\sigma_{L}}{w_{L}} \operatorname{arctanh}\left(\frac{w_{L} \tilde{x}}{\tilde{y}-\sigma_{L} \tilde{x}}\right),
$$
where $(\tilde{x}, \tilde{y})=\left(x-\bar{x}_{L}, y-\bar{y}_{L}\right)$ are relative coordinates to $e_{L}$, as given in $(2)$.

(a.2) When $L<0$, the invariant manifolds of the real saddle intersect the line $x=-u$ at the points $\left(-u, P_{L}^{ \pm}\right)$and such orbits enter the region $-u<x<v$ (one forward and the other backwards in time) intersecting the line $x=v$ at the points $\left(v, Q_{L}^{ \pm}\right)$, where

$$
P_{L}^{ \pm}=\frac{u}{\sigma_{L} \mp w_{L}}, \quad Q_{L}^{ \pm}=\mp \sqrt{\left(P_{L}^{ \pm}\right)^{2}+u^{2}-v^{2}},
$$

so that $P_{L}^{+}<0<P_{L}^{-}$and $Q_{L}^{+}<0<Q_{L}^{-}$. A first integral for the orbits between such invariant manifolds, when restricted to the region $x \leq-u$, is the function

$$
H_{L}^{S}(\tilde{x}, \tilde{y})=\log \sqrt{w_{L}^{2} \tilde{x}^{2}-\left(\tilde{y}-\sigma_{L} \tilde{x}\right)^{2}}+\frac{\sigma_{L}}{w_{L}} \operatorname{arctanh}\left(\frac{\tilde{y}-\sigma_{L} \tilde{x}}{w_{L} \tilde{x}}\right),
$$

where $(\tilde{x}, \tilde{y})=\left(x-\bar{x}_{L}, y-\bar{y}_{L}\right)$ are relative coordinates to $e_{L}$, as given in $(2)$.

(b) If $T_{L}^{2}<4 L$ and we take $2 \sigma_{L}=T_{L}$ and $\omega_{L}>0$ such that $4 \omega_{L}^{2}=4 L-T_{L}^{2}$, then the equilibrium point $e_{L}$ is an attractive virtual focus. A first integral for the orbits restricted to the region $x \leq-u$, is the function

$$
H_{L}^{F}(\tilde{x}, \tilde{y})=\log \sqrt{\left(\tilde{y}-\sigma_{L} \tilde{x}\right)^{2}+\omega_{L}^{2} \tilde{x}^{2}}-\frac{\sigma_{L}}{\omega_{L}} \arctan \left(\frac{\tilde{y}-\sigma_{L} \tilde{x}}{\omega_{L} \tilde{x}}\right),
$$

where $(\tilde{x}, \tilde{y})=\left(x-\bar{x}_{L}, y-\bar{y}_{L}\right)$ are relative coordinates to $e_{L}$, as given in (2).

A similar result holds for the dynamics associated to $e_{R}$.

Proposition 3. Consider the differential systems (1) with $0<v<u, T_{C}=0$ and $T_{R}>0$. The following statements hold. 
(a) If $T_{R}^{2}>4 R$ and we take $2 \sigma_{R}=T_{R}$ and $w_{R}>0$ such that $4 w_{R}^{2}=T_{R}^{2}-4 R$, then the equilibrium point $e_{R}$ is a repulsive virtual node when $R>0$ and $a$ real saddle for $R<0$.

(a.1) When $R>0$, the invariant manifolds of the virtual node intersect the line $x=v$ at the points $\left(v, P_{R}^{ \pm}\right)$and such orbits enter the region $-u<x<v$ backwards in time intersecting the line $x=-u$ at the points $\left(-u, Q_{R}^{ \pm}\right)$, where

$$
P_{R}^{ \pm}=-\frac{v}{\sigma_{R} \mp w_{R}}, \quad Q_{R}^{ \pm}=-\sqrt{\left(P_{R}^{ \pm}\right)^{2}+v^{2}-u^{2}},
$$

so that $P_{R}^{+}<P_{R}^{-}<0$ and $Q_{R}^{+}<Q_{R}^{-}<0$, where it is assumed $\left(P_{R}^{-}\right)^{2} \geq u^{2}-v^{2}$. A first integral for the orbits above such invariant manifolds, when restricted to the region $x \leq v$, is the function

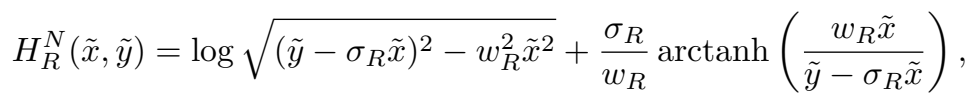
where $(\tilde{x}, \tilde{y})=\left(x-\bar{x}_{R}, y-\bar{y}_{R}\right)$ are relative coordinates to $e_{R}$, as given in $(2)$.

(a.2) When $R<0$, the invariant manifolds of the real saddle intersect the line $x=v$ at the points $\left(v, P_{R}^{ \pm}\right)$and such orbits enter the region $-u<$ $x<v$ (one forward and the other backwards in time) intersecting the line $x=-u$ at the points $\left(-u, Q_{R}^{ \pm}\right)$, where

$$
P_{R}^{ \pm}=-\frac{v}{\sigma_{R} \mp w_{R}}, \quad Q_{R}^{ \pm}= \pm \sqrt{\left(P_{R}^{ \pm}\right)^{2}+u^{2}-v^{2}},
$$

so that $P_{R}^{-}<0<P_{R}^{+}$and $Q_{R}^{-}<0<Q_{R}^{+}$. A first integral for the orbits between such invariant manifolds, when restricted to the region $x \leq v$, is the function

$$
H_{R}^{S}(\tilde{x}, \tilde{y})=\log \sqrt{w_{R}^{2} \tilde{x}^{2}-\left(\tilde{y}-\sigma_{R} \tilde{x}\right)^{2}}+\frac{\sigma_{R}}{w_{R}} \operatorname{arctanh}\left(\frac{\tilde{y}-\sigma_{R} \tilde{x}}{w_{R} \tilde{x}}\right),
$$

where $(\tilde{x}, \tilde{y})=\left(x-\bar{x}_{R}, y-\bar{y}_{R}\right)$ are relative coordinates to $e_{R}$, as given in $(2)$.

(b) If $T_{R}^{2}<4 R$ and we take $2 \sigma_{R}=T_{R}$ and $\omega_{R}>0$ such that $4 \omega_{R}^{2}=4 R-T_{R}^{2}$, then the equilibrium point $e_{R}$ is an repulsive virtual focus. A first integral for the orbits restricted to the region $x \leq-u$, is the function

$$
H_{R}^{F}(\tilde{x}, \tilde{y})=\log \sqrt{\left(\tilde{y}-\sigma_{R} \tilde{x}\right)^{2}+\omega_{R}^{2} \tilde{x}^{2}}-\frac{\sigma_{R}}{\omega_{R}} \arctan \left(\frac{\tilde{y}-\sigma_{R} \tilde{x}}{\omega_{R} \tilde{x}}\right),
$$

where $(\tilde{x}, \tilde{y})=\left(x-\bar{x}_{R}, y-\bar{y}_{R}\right)$ are relative coordinates to $e_{R}$, as given in (2).

In what follows $\mathrm{F}, \mathrm{N}$ and $\mathrm{S}$ denote a virtual focus, a virtual node and a real saddle, and the notation FN denotes that on the left hand zone we have a virtual focus and on the right hand zone we have a virtual node. Similarly for any other combinations of two letters from $\{F, N, S\}$.

Our main result is the following.

Theorem 4. For the differential systems (1) with $0<v<u, T_{R}>0, T_{L}<0$, fulfilling one of the following sets of conditions, 
(FF) $L, R>0, T_{R}<2 \sqrt{R},\left|T_{L}\right|<2 \sqrt{L}$ and

$$
\frac{T_{L}}{\sqrt{L}}+\frac{T_{R}}{\sqrt{R}}<0,
$$

see Figure 2;

(NF) $L, R>0, T_{R}<2 \sqrt{R}$, and $\left|T_{L}\right|>2 \sqrt{L}$, see Figure 3;

(NN) $L, R>0, T_{R}>2 \sqrt{R},\left|T_{L}\right|>2 \sqrt{L}$, and $Q_{L}^{+}>P_{R}^{-}$, see Figure 4;

(SF) $L<0, R>0, T_{R}<2 \sqrt{R}$ and

$$
H_{R}^{F}\left(v-\bar{x}_{R}, Q_{L}^{+}-\bar{y}_{R}\right)<H_{R}^{F}\left(v-\bar{x}_{R}, Q_{L}^{-}-\bar{y}_{R}\right),
$$

where $Q_{L}^{ \pm}$are as in (5) and $H_{R}^{F}$ as in (12), see Figure 5;

(FS) $L>0, R<0,\left|T_{L}\right|<2 \sqrt{L}$ and

$$
H_{L}^{F}\left(-u-\bar{x}_{L}, Q_{R}^{+}-\bar{y}_{L}\right)<H_{L}^{F}\left(-u-\bar{x}_{L}, Q_{R}^{-}-\bar{y}_{L}\right),
$$

where $Q_{R}^{ \pm}$are as in (10) and $H_{L}^{F}$ as in (7), see Figure 6;

(SN) $L<0, R>0, T_{R}>2 \sqrt{R}, Q_{L}^{+}>P_{R}^{-}$and

$$
H_{R}^{N}\left(v-\bar{x}_{R}, Q_{L}^{+}-\bar{y}_{R}\right)<H_{R}^{N}\left(v-\bar{x}_{R}, Q_{L}^{-}-\bar{y}_{R}\right),
$$

where $Q_{L}^{ \pm}$are as in (5) and $H_{R}^{N}$ as in (9), see Figure 7;

(NS) $L>0, R<0,\left|T_{L}\right|>2 \sqrt{L}$ and

$$
H_{L}^{N}\left(-u-\bar{x}_{L}, Q_{R}^{+}-\bar{y}_{L}\right)<H_{L}^{N}\left(-u-\bar{x}_{L}, Q_{R}^{-}-\bar{y}_{L}\right),
$$

where $Q_{R}^{ \pm}$are as in (10) and $H_{L}^{N}$ as in (4), see Figure 8, and condition (17) is automatically fulfilled if $P_{L}^{+}>Q_{R}^{-}$;

(SS) $L<0, R<0, Q_{L}^{+}>P_{R}^{-}$and

$$
H_{R}^{S}\left(v-\bar{x}_{R}, Q_{L}^{+}-\bar{y}_{R}\right)>H_{R}^{S}\left(v-\bar{x}_{R}, Q_{L}^{-}-\bar{y}_{R}\right),
$$

where $Q_{L}^{ \pm}$are as in (5), $P_{R}^{ \pm}$as in (10) and $H_{R}^{S}$ as in (11), see Figure 9, and condition (18) is automatically fulfilled if $P_{R}^{+}<Q_{L}^{-}$;

the following statements hold.

(a) If $T_{C}=0$ then the origin is surrounded by a bounded period annulus whose most external periodic orbit, which is tangent to the straight line $x=v$, in its outerpart is unstable. There exists also a stable limit cycle intersecting the three zones surrounding such period annulus.

(b) There exists $\varepsilon>0$ such that if $-\varepsilon<T_{C}<0$ the origin is surrounded by at least two limit cycles, the smaller is unstable and the bigger is stable.

The proof of Theorem 4 is given in section 4 where we have separated in different subsections its proof, for each one of the different conditions given in the theorem.

Different Poincaré discs, coming from the compactification of the phase portrait, illustrating the eight condition sets where Theorem 4 applies are drawn in Figures 2-9. We have choosen $1=v<u=2$ in all the cases, being $T_{L}, T_{R}, L$ and $R$ as given in Table 1.

After reversing the time and/or a change of variables that interchanges the left and the right zones (when needed), we can write similar results to the ones given in Theorem 4 for different hypotheses, as for instance, when $0<u<v$ or when $T_{L}>0$ and $T_{R}<0$ or both. Their precise statements and proofs are direct consequences of the one of Theorem 4 and they will not be provided. 


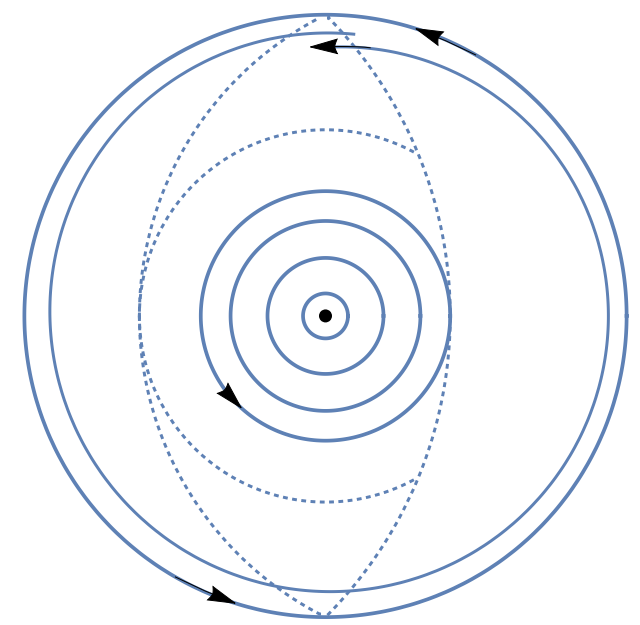

Figure 2. Poincaré disc corresponding to the FF case when $T_{C}=$ 0 and the hypotheses of Theorem $4(\mathrm{FF})$ are satisfied, so that the periodic orbit at infinity is repulsive. Any nearby orbit allows to build a positively invariant compact set. Note that the vertical lines $x=-u$ and $x=v$ appear as arcs of circles connecting the north and south poles due to the compactification.

TABle 1. Numerical values chosen for drawing the Poincaré discs.

\begin{tabular}{|c|c|c|c|c|c|}
\hline Figure & Case & $T_{L}$ & $L$ & $T_{R}$ & $R$ \\
\hline 2 & $\mathrm{FF}$ & -2 & 1.49 & 0.2 & 0.05 \\
\hline 3 & $\mathrm{NF}$ & -2 & 0.75 & 0.2 & 0.05 \\
\hline 4 & $\mathrm{NN}$ & -2 & 0.51 & 0.4 & 0.03 \\
\hline 5 & $\mathrm{SF}$ & -2 & -1.25 & 0.3 & 1.0225 \\
\hline 6 & $\mathrm{FS}$ & -2 & 1.49 & 0.1 & -0.06 \\
\hline 7 & $\mathrm{SN}$ & -2 & -1.25 & 0.3 & 0.0125 \\
\hline 8 & $\mathrm{NS}$ & -0.6 & 0.05 & 0.1 & -0.06 \\
\hline 9 & $\mathrm{SS}$ & -1 & -1.25 & 0.16 & -0.0561 \\
\hline
\end{tabular}

Note that in Theorem 4 the case (FN) is not considered, since under the assumed hypotheses it is not possible to build a positively invariant compact set containing the period annulus in its interior. In fact, Theorem 4 relies in the fact that when such a compact set exists we must also conclude the existence of a stable limit cycle surrounding the period annulus that appears for $T_{C}=0$, that is not possible in the (FN) case, see below Remark 14 .

Let us define the set

$$
S_{\mathrm{RVF}}=\mathbb{R}^{2} \backslash\left\{(x, y) \in \mathbb{R}^{2}: x g(x)<0\right\} .
$$

Note that $x g(x)<0$ only in two situations, which can arise separately or not, namely when there are real equilibria of saddle type. Thus, if $L<0$ then $x g(x)<0$ for $x<\bar{x}_{L}=-u(1-1 / L)<-u$, while if $R<0$ then $x g(x)<0$ for $x>\bar{x}_{R}=$ $v(1-1 / R)>v$. Therefore, $S_{\mathrm{RVF}}$ is indeed the whole plane when $L>0, R>0$. 


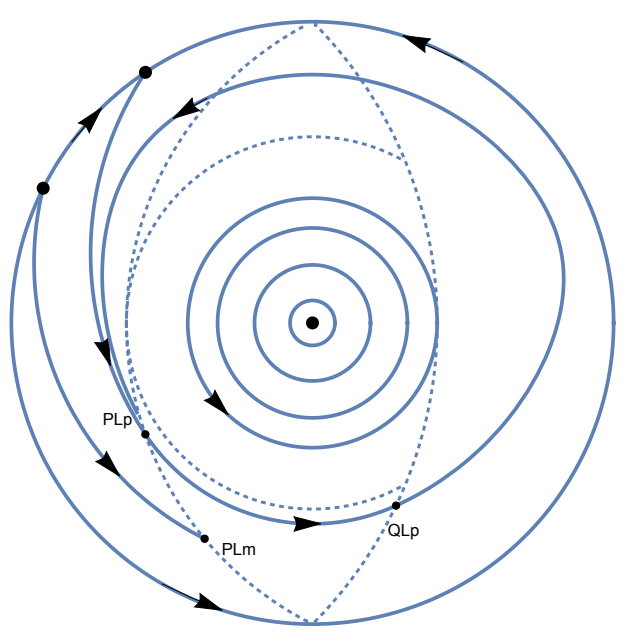

Figure 3. Poincaré disc corresponding to the NF case when $T_{C}=$ 0 . No additional hypotheses are required to get a compact positive invariant set containing the period annulus. We indicate only the values of ordinates, so that $P_{L}^{-}$and $P_{L}^{+}$are the ordinates of the intersection points for the invariant manifolds of the saddle $e_{L}$ with the straight line $x=-u$, and $Q_{L}^{-}$(not shown) and $Q_{L}^{+}$are their intersections with $x=v$.

Clearly, the two limit cycles predicted in Theorem 4 are located within such a set, which excludes any possible real saddle. Precisely, another important remark for system (1) is its character of being a rotated vector field whithin $S_{\mathrm{RVF}}$ with respect to the parameter $T_{C}$, see $[32,33]$. Effectively, the determinant

$$
\left|\begin{array}{cc}
F(x)-y & g(x) \\
\frac{\partial}{\partial T_{C}}(F(x)-y) & \frac{\partial}{\partial T_{C}} g(x)
\end{array}\right|=\left|\begin{array}{cc}
F(x)-y & g(x) \\
\frac{\partial}{\partial T_{C}} F(x) & 0
\end{array}\right|=-g(x) \frac{\partial}{\partial T_{C}} F(x)
$$

does not change its sign in $S_{\mathrm{RVF}}$, since the expression

$$
g(x) \frac{\partial}{\partial T_{C}} F(x)= \begin{cases}-u g(x) & \text { if } x \leq-u, \\ x g(x) & \text { if }-u \leq x \leq v, \\ v g(x) & \text { if } x \geq v\end{cases}
$$

is non-negative there. Rotated vector fields have the non-intersection property, that is, closed trajectories of the system for two different values of the distinguished parameter $T_{C}$ cannot intersect. As a consequence, we can assure that the unstable limit cycle growths in size as long as $\left(-T_{C}\right)$ increases, while simultaneously the stable limit cycle shrinks, so that both limit cycles approach one another. In fact, they may collapse for a certain value $T_{C}^{*}<0$ in a semi-stable limit cycle, to disappear for bigger values of $\left(-T_{C}\right)$. Our last result indicates that this is indeed the case.

Theorem 5. For the differential systems (1) with $0<v<u, T_{R}>0, T_{L}<0$, fulfilling any of the sets of conditions of Theorem 4, there exists a value $T_{C}^{*}<0$ such that there are no limit cycles for $T_{C}<T_{C}^{*}$. 


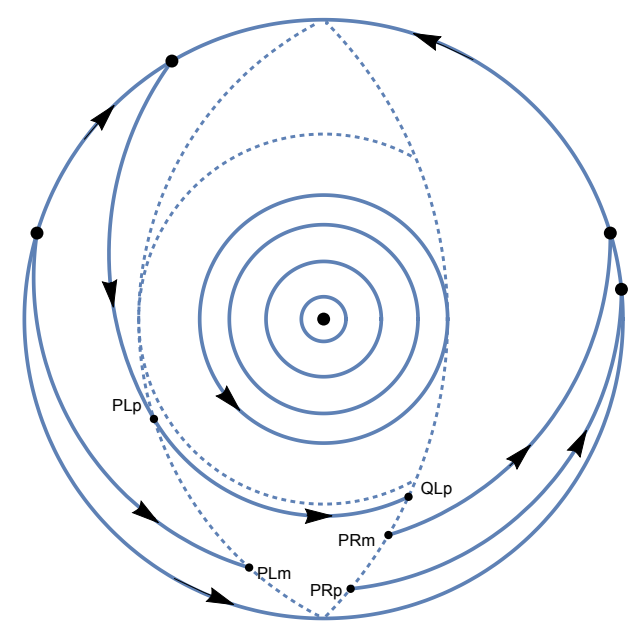

Figure 4. Poincaré disc corresponding to the NN case when $T_{C}=$ 0 and the hypotheses of Theorem $4(\mathrm{NN})$ are satisfied. We indicate only the values of ordinates, so that $P_{L}^{-}$and $P_{L}^{+}$are the ordinates of the intersection points for the invariant manifolds of the virtual node $e_{L}$ with the straight line $x=-u$, and $Q_{L}^{-}$(not shown) and $Q_{L}^{+}$are their intersections with $x=v$. Similarly, $P_{R}^{-}$and $P_{R}^{+}$are the ordinates of the intersection points for the invariant manifolds of the virtual node $e_{R}$ with the straight line $x=v$.

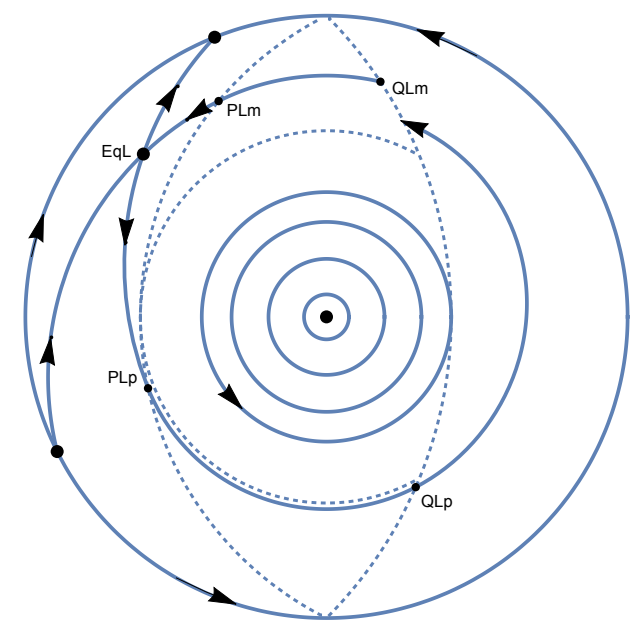

Figure 5. Poincaré disc corresponding to the SF case when $T_{C}=$ 0 and the hypotheses of Theorem 4 (SF) are satisfied.

The proof of Theorem 5 is given in Section 5. The computation for each case of the greatest lower bound for the value $T_{C}^{*}$ predicted in Theorem 5 , which will correspond with a saddle-node bifurcation of periodic orbits, is beyond the scope 


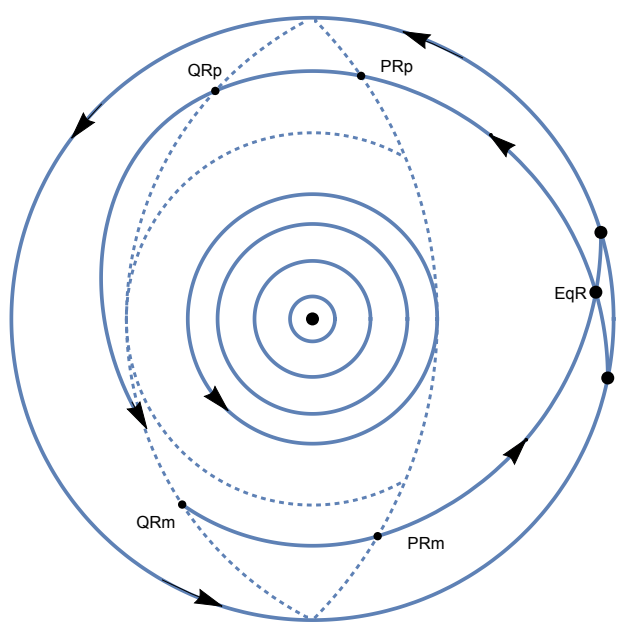

Figure 6. Poincaré disc corresponding to the $\mathrm{FS}$ case when $T_{C}=$ 0 and the hypotheses of Theorem 4 (FS) are satisfied.

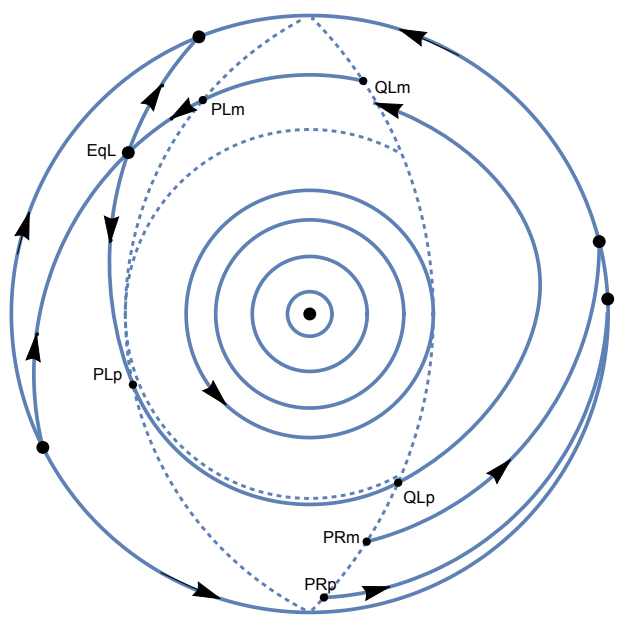

Figure 7. Poincaré disc corresponding to the $\mathrm{SN}$ case when $T_{C}=$ 0 and the hypotheses of Theorem $4(\mathrm{SN})$ are satisfied.

of this paper. Such global bifurcations are rather difficult to obtain by analytical techniques and typically one must resort to numerical methods.

The remaining sections are organized as follows. First, we include in Section 2 some auxiliary results to be later needed. In Section 3, we compute the distinguished points and the first integrals that appear in the statements of Theorem 4, which is proved in Section 4. To finish, Section 5 is devoted to the proof of Theorem 5.

\section{Preliminary Results}

We will need the following result whose proof is given in [5]. 


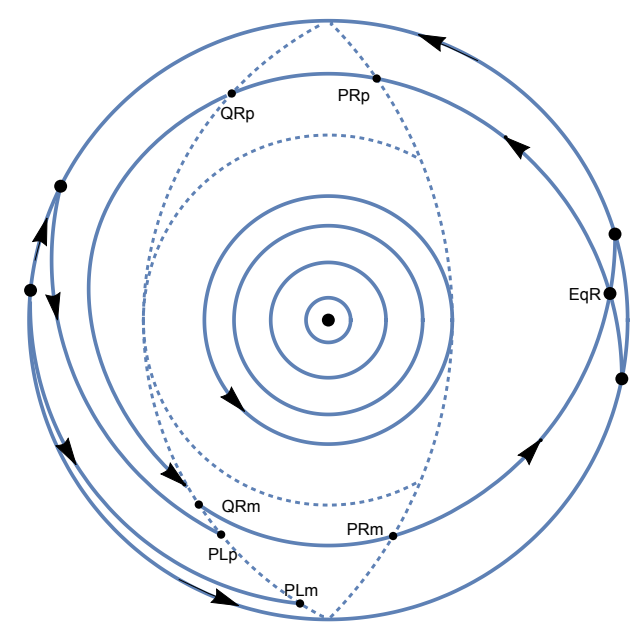

Figure 8. Poincaré disc corresponding to the NS case when $T_{C}=$ 0 and the hypotheses of Theorem 4 (NS) are satisfied. If $P_{L}^{+}>Q_{R}^{-}$ then the existence of a compact positive invariant set including the period annulus is guaranteed.

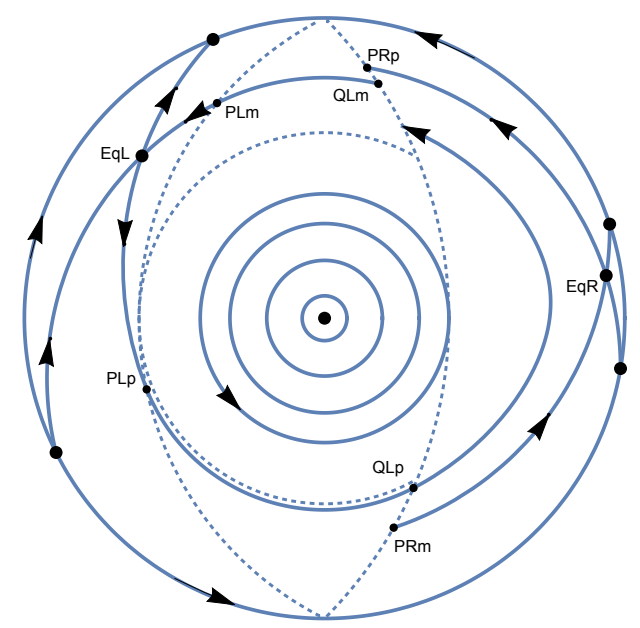

Figure 9. Poincaré disc corresponding to the SS case when $T_{C}=$ 0 and the hypotheses of Theorem 4 (SS) are satisfied. If $P_{R}^{+}<Q_{L}^{-}$ then the existence of a compact positive invariant set including the period annulus is guaranteed.

Proposition 6. Let $x(t)$ be a periodic solution of period $T$ of the planar differential system $\dot{x}=f(x)$, and let

$$
\mathcal{I}=\int_{0}^{T} \text { divergence }\left.(f(x))\right|_{x=x(t)} d t .
$$


If $\mathcal{I}>0$ then $x(t)$ is an unstable hyperbolic limit cycle, and if $\mathcal{I}<0$ then $x(t)$ is a stable hyperbolic limit cycle.

The instability of the outermost periodic orbit for the bounded center that appears for $T_{C}=0$ under the hypothesis of the reference case, and the bifurcation of a unstable limit cycle from it when $T_{C}<0$, are shown next.

Proposition 7. Consider the differential systems (1) and assume $0<v<u$, $T_{C}=0$ and $T_{R}>0$. Then the origin is surrounded by a bounded period annulus whose most external periodic orbit, which is tangent to the line $x=v$, is in its outerpart unstable.

Proof. Since $T_{C}=0$ and $v<u$, we have a circular period annulus tangent to the line $x=v$ and totally contained in the band $-u<-v \leq x \leq v$. The most external periodic orbit, which is tangent to the line $x=v$ passes through the point $(v, 0)$. Recalling that the dynamics in the right hand zone $x>v$ is given by

$$
\dot{x}=T_{R}(x-v)-y, \quad \dot{y}=R(x-v)+v,
$$

we conclude that

$$
\left.\ddot{x}\right|_{(x, y)=(v, 0)}=\left[T_{R}\left(T_{R}(x-v)-y\right)-(R(x-v)+v)\right]_{(x, y)=(v, 0)}=-v<0,
$$

so that we can define a local return map with respect to the straight line $x=v$. More precisely, if we take an orbit starting at the point $\left(v, y_{0}\right)$ with $y_{0}<0$ and small in absolute value, then is it assured that such an orbit enters the half-plane $x>v$ and returns to the straight line $x=v$ at a point $\left(v, y_{1}\right)$ with $y_{1}>0$. In fact, we have the expansion

$$
y_{1}=-y_{0}+\frac{2 T_{R}}{3 v} y_{0}^{2}+O\left(y_{0}^{3}\right),
$$

see for instance Proposition 8 in [11]. Consequently, as $T_{R}>0$ we get $y_{1}>\left|y_{0}\right|$ and the proof is complete.

Proposition 8. Consider differential systems (1) and assume $0<v<u, T_{R}>0$, and $T_{L}<0$. Taking $T_{C}$ as a bifurcation parameter, for $T_{C}=0$ the system undergoes a focus-center-limit cycle bifurcation, so that there exists $\varepsilon>0$ sufficiently small such that for $-\varepsilon<T_{C}<0$ an unstable limit cycle bifurcates from the period annulus.

Proof. In view of Proposition 7 when $T_{C}=0$ the most external periodic orbit, which is tangent to the line $x=v$ is in its outerpart unstable.

Now if we perturb system (1) with $T_{C}=0$ by taking $T_{C}<0$ and sufficiently small, then in the central zone the origin becomes a stable focus, but since the external periodic orbit was unstable, by Bendixson-Poincaré theorem (recall that in the central zone the differential system is linear), there is a periodic solution $\gamma(t)$ crossing the straight line $x=v$. It remains to show that there is an unstable limit cycle. Note that the Poincaré map defined in a segment with endpoints $(v, 0)$ and $(v+\delta, 0)$ for some $\delta>0$ is analytic (because it is the composition of two analytic maps) . Assume that $\gamma(t)$ is not a limit cycle, i.e. $\gamma(t)$ is not isolated in the set of all periodic solutions of system (1). Then by the analyticity of the Poincaré map it is the identity, in contradiction with the fact that the external periodic solution of the period annulus passing through the point $(v, 0)$ is unstable. So all the periodic 
solutions crossing the straight line $x=v$ are isolated in the set of all periodic solutions of system (1). This completes the proof.

For a quantitative characterization of the focus-center-limit cycle bifurcation of Proposition 8, which can appear in the more general setting of discontinuous systems without sliding dynamics, see [29].

\section{Notable points And first integrals for $T_{C}=0$}

Here we computing notable points and first integrals under hypotheses $0<v<u$, $T_{R}>0, T_{L}<0$, for the case of reference $T_{C}=0$. This amounts to show propositions 2 and 3 .

Proof of Proposition 2. When $T_{C}=0$, if we are in a node or saddle case, then the coordinates of the virtual or real equilibrium $e_{L}$ turn out to be

$$
e_{L}=\left(\frac{u}{\sigma_{L}^{2}-w_{L}^{2}}-u, \frac{2 \sigma_{L}}{\sigma_{L}^{2}-w_{L}^{2}} u\right),
$$

so that to find the intersection points of its invariant manifolds with the straight line $x=-u$ we must solve for $\alpha$ the linear system of equations

$$
\left(\begin{array}{c}
\frac{u}{\sigma_{L}^{2}-w_{L}^{2}}-u \\
\frac{2 \sigma_{L}}{\sigma_{L}^{2}-w_{L}^{2}} u
\end{array}\right)+\alpha\left(\begin{array}{c}
1 \\
\sigma_{L} \mp w_{L}
\end{array}\right)=\left(\begin{array}{c}
-u \\
P_{L}^{ \pm}
\end{array}\right) .
$$

We immediately obtain $\alpha=-u /\left(\sigma_{L}^{2}-w_{L}^{2}\right)$, and so

$$
P_{L}^{ \pm}=\frac{2 \sigma_{L}}{\sigma_{L}^{2}-w_{L}^{2}} u-\frac{\sigma_{L} \mp w_{L}}{\sigma_{L}^{2}-w_{L}^{2}} u=\frac{\sigma_{L} \pm w_{L}}{\sigma_{L}^{2}-w_{L}^{2}} u=\frac{u}{\sigma_{L} \mp w_{L}} .
$$

Note that for $T_{L}<0$, the node case implies $\sigma_{L}<-w_{L}<0$ so that $P_{L}^{-}<P_{L}^{+}<0$, while in the saddle case we have $-w_{L}<\sigma_{L}<0$ and therefore $P_{L}^{+}<0<P_{L}^{-}$.

In computing the first integral for non-focus cases, we translate the left system to put $e_{L}$ at the origin so that, after eliminating time, the system becomes equivalent to the homogeneous differential equation

$$
\frac{d y}{d x}=\frac{\left(\sigma_{L}^{2}-w_{L}^{2}\right) x}{2 \sigma_{L} x-y},
$$

which after the substitution $y=s x$ becomes

$$
\frac{d s}{d x}=\frac{\left(s-\sigma_{L}\right)^{2}-w_{L}^{2}}{\left(2 \sigma_{L}-s\right) x} .
$$

Separating variables, we can write the decomposition

$$
\frac{\left(\sigma_{L}-w_{L}\right) d s}{2 w_{L}\left(s-\sigma_{L}-w_{L}\right)}-\frac{\left(\sigma_{L}+w_{L}\right) d s}{2 w_{L}\left(s-\sigma_{L}+w_{L}\right)}=\frac{d x}{x} .
$$

Before making the integration, we note that in the node case we need to work, regarding the point $e_{L}$ once assumed to be in the origin, in the region with $x<0$ and

what leads to

$$
s=\frac{y}{x}<\sigma_{L}-w_{L}<\sigma_{L}+w_{L}<0,
$$

$$
\frac{\left(\sigma_{L}-w_{L}\right)}{2 w_{L}} \log \left[-\left(s-\sigma_{L}-w_{L}\right)\right]-\frac{\left(\sigma_{L}+w_{L}\right)}{2 w_{L}} \log \left[-\left(s-\sigma_{L}+w_{L}\right)\right]=\log [-x]+C .
$$


We write

$$
\frac{\sigma_{L}}{2 w_{L}} \log \frac{s-\sigma_{L}-w_{L}}{s-\sigma_{L}+w_{L}}-\frac{1}{2} \log \left[\left(s-\sigma_{L}-w_{L}\right)\left(s-\sigma_{L}+w_{L}\right)\right]-\log [-x]=C,
$$

and so a relevant first integral for the node case is

$$
\frac{\sigma_{L}}{2 w_{L}} \log \frac{y-\sigma_{L} x-w_{L} x}{y-\sigma_{L} x+w_{L} x}-\frac{1}{2} \log \left[\left(y-\sigma_{L} x\right)^{2}-w_{L}^{2} x^{2}\right],
$$

or equivalently

$$
-\frac{\sigma_{L}}{2 w_{L}} \log \frac{y-\sigma_{L} x+w_{L} x}{y-\sigma_{L} x-w_{L} x}-\frac{1}{2} \log \left[\left(y-\sigma_{L} x\right)^{2}-w_{L}^{2} x^{2}\right] .
$$

We choose as first integral the more compact expression

$$
H_{L}^{N}(x, y)=\log \sqrt{\left(y-\sigma_{L} x\right)^{2}-w_{L}^{2} x^{2}}+\frac{\sigma_{L}}{w_{L}} \operatorname{arctanh}\left(\frac{w_{L} x}{y-\sigma_{L} x}\right) .
$$

In the saddle case we need to work, assuming the point $e_{L}$ to be at the origin, in the region with $x>0$ and

$$
\sigma_{L}-w_{L}<s=\frac{y}{x}<\sigma_{L}+w_{L}
$$

what leads to

$$
\frac{\left(\sigma_{L}-w_{L}\right)}{2 w_{L}} \log \left[-\left(s-\sigma_{L}-w_{L}\right)\right]-\frac{\left(\sigma_{L}+w_{L}\right)}{2 w_{L}} \log \left(s-\sigma_{L}+w_{L}\right)=\log (x)+C .
$$

We write

$$
\frac{\sigma_{L}}{2 w_{L}} \log \frac{w_{L}+\sigma_{L}-s}{w_{L}-\sigma_{L}+s}-\frac{1}{2} \log \left[\left(w_{L}+\sigma_{L}-s\right)\left(w_{L}-\sigma_{L}+s\right)\right]-\log (x)=C,
$$

and then a relevant first integral for the saddle case is

$$
-\frac{\sigma_{L}}{2 w_{L}} \log \frac{w_{L} x-\sigma_{L} x+y}{w_{L} x+\sigma_{L} x-y}-\frac{1}{2} \log \left[w_{L}^{2} x^{2}-\left(y-\sigma_{L} x\right)^{2}\right]
$$

and we take

$$
H_{L}^{S}(x, y)=\log \sqrt{w_{L}^{2} x^{2}-\left(y-\sigma_{L} x\right)^{2}}+\frac{\sigma_{L}}{w_{L}} \operatorname{arctanh}\left(\frac{y-\sigma_{L} x}{w_{L} x}\right) .
$$

In computing the first integral for the focus case, we note first that now

$$
e_{L}=\left(\frac{u}{\sigma_{L}^{2}+\omega_{L}^{2}}-u, \frac{2 \sigma_{L}}{\sigma_{L}^{2}+\omega_{L}^{2}} u\right),
$$

so that translating coordinates to put $e_{L}$ at the origin and eliminating time, the system becomes equivalent to the homogeneous differential equation

$$
\frac{d y}{d x}=\frac{\left(\sigma_{L}^{2}+\omega_{L}^{2}\right) x}{2 \sigma_{L} x-y} .
$$

After the substitution $y=s x$, it becomes

$$
\frac{d s}{d x}=\frac{\left(s-\sigma_{L}\right)^{2}+\omega_{L}^{2}}{\left(2 \sigma_{L}-s\right) x},
$$

which can be decomposed into

$$
\frac{\sigma_{L}-s}{\left(s-\sigma_{L}\right)^{2}+\omega_{L}^{2}} d s+\frac{\sigma_{L}}{\left(s-\sigma_{L}\right)^{2}+\omega_{L}^{2}} d s=\frac{d x}{x} .
$$


Taking into account that we are interested in the left part with respect to the focus, we assume $x<0$ to integrate the above expression, so that we write

$$
-\frac{1}{2} \log \left[\left(s-\sigma_{L}\right)^{2}+\omega_{L}^{2}\right]+\frac{\sigma_{L}}{\omega_{L}} \arctan \left(\frac{s-\sigma_{L}}{\omega_{L}}\right)=\log [-x]+C,
$$

leading to the first integral

$$
H_{L}^{F}(x, y)=\log \sqrt{\left(y-\sigma_{L} x\right)^{2}+\omega_{L}^{2} x^{2}}-\frac{\sigma_{L}}{\omega_{L}} \arctan \left(\frac{y-\sigma_{L} x}{\omega_{L} x}\right) .
$$

Proof of Proposition 3. The procedure is totally analogous. For $T_{C}=0$, in the node or saddle cases, the coordinates of the virtual or real equilibrium $e_{R}$ turn out to be

$$
e_{R}=\left(v-\frac{v}{\sigma_{R}^{2}-w_{R}^{2}},-\frac{2 \sigma_{R}}{\sigma_{R}^{2}-w_{R}^{2}} v\right),
$$

so that to compute $P_{R}^{ \pm}$we must solve for $\alpha$ the linear system of equations

$$
\left(\begin{array}{c}
v-\frac{v}{\sigma_{R}^{2}-w_{R}^{2}} \\
-\frac{2 \sigma_{R}}{\sigma_{R}^{2}-w_{R}^{2}} v
\end{array}\right)+\alpha\left(\begin{array}{c}
1 \\
\sigma_{R} \mp w_{R}
\end{array}\right)=\left(\begin{array}{c}
v \\
P_{R}^{ \pm}
\end{array}\right) .
$$

We immediately obtain $\alpha=v /\left(\sigma_{R}^{2}-w_{R}^{2}\right)$, and so

$$
P_{R}^{ \pm}=-\frac{2 \sigma_{R}}{\sigma_{R}^{2}-w_{R}^{2}} v+\frac{\sigma_{R} \mp w_{R}}{\sigma_{R}^{2}-w_{R}^{2}} v=-\frac{\sigma_{R} \pm w_{R}}{\sigma_{R}^{2}-w_{R}^{2}} u=-\frac{v}{\sigma_{R} \mp w_{R}} .
$$

Note that under the assumption $T_{R}>0$, the node case implies $0<w_{R}<\sigma_{R}$ so that $P_{R}^{+}<P_{R}^{-}<0$, while in the saddle case we have $0<\sigma_{R}<w_{R}$ and therefore $P_{R}^{-}<0<P_{R}^{+}$.

For the node and saddle cases, we make a translation of the right system to put $e_{R}$ at the origin, to get the homogeneous differential equation

$$
\frac{d y}{d x}=\frac{\left(\sigma_{R}^{2}-w_{R}^{2}\right) x}{2 \sigma_{R} x-y},
$$

which after the substitution $y=s x$ becomes

$$
\frac{d s}{d x}=\frac{\left(s-\sigma_{R}\right)^{2}-w_{R}^{2}}{\left(2 \sigma_{R}-s\right) x} .
$$

Separating variables, we now have

$$
\frac{\left(\sigma_{R}-w_{R}\right) d s}{2 w_{R}\left(s-\sigma_{R}-w_{R}\right)}-\frac{\left(\sigma_{R}+w_{R}\right) d s}{2 w_{R}\left(s-\sigma_{R}+w_{R}\right)}=\frac{d x}{x} .
$$

We note that in the node case we need to work, regarding the point $e_{R}$ once assumed to be in the origin, in the region with $x>0$ and

$$
0<\sigma_{R}-w_{R}<\sigma_{R}+w_{R}<s=\frac{y}{x},
$$

what leads to

$$
\frac{\left(\sigma_{R}-w_{R}\right)}{2 w_{R}} \log \left(s-\sigma_{R}-w_{R}\right)-\frac{\left(\sigma_{R}+w_{R}\right)}{2 w_{R}} \log \left(s-\sigma_{R}+w_{R}\right)=\log (x)+C .
$$

We write

$$
-\frac{1}{2} \log \left[\left(s-\sigma_{R}+w_{R}\right)\left(s-\sigma_{R}-w_{R}\right)\right]-\frac{\sigma_{R}}{2 w_{R}} \log \frac{s-\sigma_{R}+w_{R}}{s-\sigma_{R}-w_{R}}-\log (x)=C,
$$


and so a relevant first integral for the node case is

$$
H_{R}^{N}(x, y)=\log \sqrt{\left(y-\sigma_{R} x\right)^{2}-w_{R}^{2} x^{2}}+\frac{\sigma_{R}}{w_{R}} \operatorname{arctanh}\left(\frac{w_{R} x}{y-\sigma_{R} x}\right) .
$$

In the saddle case we need to work, assuming the point $e_{R}$ to be at the origin, in the region with $x<0$ and

$$
\sigma_{R}-w_{R}<s=\frac{y}{x}<\sigma_{R}+w_{R}
$$

what leads to

$$
\frac{\left(\sigma_{R}-w_{R}\right)}{2 w_{R}} \log \left[-\left(s-\sigma_{R}-w_{R}\right)\right]-\frac{\left(\sigma_{R}+w_{R}\right)}{2 w_{R}} \log \left[\left(s-\sigma_{R}+w_{R}\right)\right]=\log [-x]+C .
$$

We write

$$
\frac{\sigma_{R}}{2 w_{R}} \log \frac{w_{R}+\sigma_{R}-s}{w_{R}-\sigma_{R}+s}-\frac{1}{2} \log \left[\left(w_{R}-\sigma_{R}+s\right)\left(w_{R}+\sigma_{R}-s\right)\right]-\log [-x]=C,
$$

and then a relevant first integral for the saddle case is

$$
H_{R}^{S}(x, y)=\log \sqrt{w_{R}^{2} x^{2}-\left(y-\sigma_{R} x\right)^{2}}+\frac{\sigma_{R}}{w_{R}} \operatorname{arctanh}\left(\frac{y-\sigma_{R} x}{w_{R} x}\right) .
$$

In computing the first integral for the focus case, we note first that now

$$
e_{R}=\left(v-\frac{v}{\sigma_{R}^{2}+\omega_{R}^{2}},-\frac{2 \sigma_{L}}{\sigma_{R}^{2}+\omega_{R}^{2}} v\right)
$$

so that translating coordinates to put $e_{R}$ at the origin and eliminating time, the system becomes equivalent to the homogeneous differential equation

$$
\frac{d y}{d x}=\frac{\left(\sigma_{R}^{2}+\omega_{R}^{2}\right) x}{2 \sigma_{R} x-y} .
$$

After the substitution $y=s x$, it becomes

$$
\frac{d s}{d x}=\frac{\left(s-\sigma_{R}\right)^{2}+\omega_{R}^{2}}{\left(2 \sigma_{R}-s\right) x},
$$

which can be decomposed into

$$
\frac{\sigma_{R}-s}{\left(s-\sigma_{R}\right)^{2}+\omega_{R}^{2}} d s+\frac{\sigma_{R}}{\left(s-\sigma_{R}\right)^{2}+\omega_{R}^{2}} d s=\frac{d x}{x} .
$$

Taking into account that we are here interested in the right part with respect to the focus, we assume $x>0$ to integrate the above expression, so that we write

$$
-\frac{1}{2} \log \left[\left(s-\sigma_{R}\right)^{2}+\omega_{R}^{2}\right]+\frac{\sigma_{R}}{\omega_{R}} \arctan \left(\frac{s-\sigma_{R}}{\omega_{R}}\right)=\log (x)+C,
$$

leading to the first integral

$$
H_{R}^{F}(x, y)=\log \sqrt{\left(y-\sigma_{R} x\right)^{2}+\omega_{R}^{2} x^{2}}-\frac{\sigma_{R}}{\omega_{R}} \arctan \left(\frac{y-\sigma_{R} x}{\omega_{R} x}\right) .
$$




\section{Proof of Theorem 4}

We first note that in view of Proposition 7 when $T_{C}=0$ we have a circular period annulus tangent to the line $x=v$, totally contained in the band $-u<-v \leq x \leq v$ and the outermost periodic orbit of the annulus is unstable. Furthermore, in view of Proposition 8 if we perturb this situation by taking $T_{C}<0$ and sufficiently small, then there is a bifurcation of an unstable limit cycle from the period annulus.

In order to complete the proof of Theorem 4 we will show that in each of its eight assumptions there exists a positively invariant set $\Omega$ homeomorphic to a closed disc containing the period annulus surrounding the origin. The following proposition ensures that this is enough to complete the proof of Theorem 4.

Proposition 9. Consider the Liénard piecewise linear differential system (1) with $T_{C}=0$. Assume that in each of the eight statements of Theorem 4 there exists a positively invariant compact set $\Omega$ homeomorphic to a closed disc containing the period annulus surrounding the origin. Then there is a stable limit cycle $\gamma(t)$ surrounding the mentioned period annulus.

Proof. Under the assumptions of Proposition 9 and from Proposition 7, it follows by the Bendixson-Poincaré Theorem that there is a periodic solution $\gamma(t)$ surrounding the mentioned period annulus. It remains to show that there is a stable limit cycle. Again, the Poincaré map defined on a segment with endpoints $(v, 0)$ and a point outside the region limited by $\gamma(t)$ but close to $\gamma(t)$ is analytic (because it is the composition of two or three analytic maps). The rest of the proof follows in a similar way to the last part of the proof of Proposition 8. Doing that we obtain that all the periodic solutions surrounding the period annulus are isolated in the set of all periodic solutions of system (1). Hence these periodic solutions are limit cycles. Due to the positive invariance of the set $\Omega$ minus the period annulus, at least one of these possible periodic solutions is a stable limit cycle.

Proposition 10. Assume that in each of the eight statements of Theorem 4 we are under the assumptions of Proposition 9. For $\varepsilon>0$ sufficiently small and $-\varepsilon<T_{C}<0$, there exists at least two limit cycles of the Liénard piecewise linear differential systems (1), the smallest is unstable and the biggest is stable.

Proof. We perturb system (1) with $T_{C}=0$ by taking $T_{C}<0$ and sufficiently small. Then the stable limit cycle given in Proposition 9 remains, and by Proposition 8 one unstable limit cycle appears near the region previously occupied by the period annulus. Thus this perturbed system has at least two limit cycles. This concludes the proof.

In view of Propositions 9 and 10 in order to prove Theorem 4 it only remains to show that under each of the eight statements of Theorem 4 the existence of the compact set $\Omega$ defined in Proposition 9 when $T_{C}=0$.

Proof of statement (FF) of Theorem 4. We recall that $0<v<u, T_{R}>0, T_{L}<0$ and

$$
R, L>0, T_{R}<2 \sqrt{R},\left|T_{L}\right|<2 \sqrt{L}, \frac{T_{L}}{\sqrt{4 L-T_{L}^{2}}}+\frac{T_{R}}{\sqrt{4 R-T_{R}^{2}}}<0,
$$

where the last inequality is easily deduced from condition (13), by using the monotony of the function $h(x)=x / \sqrt{4-x^{2}}$ for $x \in(-2,2)$. Note that under these assumptions system (1) has two virtual foci. We study the planar differential systems 
(1) in the Poincaré disc, see Figure 2 and the Apendix B for the definition of the Poincaré disc. Since the differential system has no singular points at infinity, the set of points at infinity becomes a closed orbit, that is, we have a periodic orbit at infinity. We will show that under the assumptions of statement (FF) this periodic orbit is unstable.

To do so, we will use Proposition 6. First, we make the change of variables

$$
x=\frac{\cos \theta}{r}, \quad y=\frac{\sin \theta}{r}, \quad \theta \in \mathbb{S}^{1}, \quad r>0 .
$$

either in the right and the left zones. On the right zone, if we denote by $\left(x_{R}, y_{R}\right)$ the old variables and by $\left(r_{R}, \theta\right)$ the new ones, where $\theta \in[-\pi / 2, \pi / 2]$ and $v r_{R} \leq \cos \theta$, we get that

$$
\begin{aligned}
\dot{r}_{R} & =-\left(x_{R} \dot{x}_{R}+y_{R} \dot{y}_{R}\right) r_{R}^{3} \\
& =-r_{R}\left\{T_{R} \cos ^{2} \theta+(R-1) \cos \theta \sin \theta-r_{R} v\left[T_{R} \cos \theta+(R-1) \sin \theta\right]\right\}, \\
\dot{\theta} & =r_{R}^{2}\left(\dot{y}_{R} x_{R}-y_{R} \dot{x}_{R}\right) \\
& =R \cos ^{2} \theta+\sin ^{2} \theta-T_{R} \cos \theta \sin \theta-r_{R} v\left[(R-1) \cos \theta-T_{R} \sin \theta\right],
\end{aligned}
$$

while on the left zone, if we denote by $\left(x_{L}, y_{L}\right)$ the old variables and by $\left(r_{L}, \theta\right)$ the new ones, where $\theta \in[\pi / 2,3 \pi / 2]$ and $-u r_{L} \leq \cos \theta$, we get that

$$
\begin{aligned}
\dot{r}_{L} & =-\left(x_{L} \dot{x}_{L}+y_{L} \dot{y}_{L}\right) r_{L}^{3} \\
& =-r_{L}\left\{T_{L} \cos ^{2} \theta+(L-1) \cos \theta \sin \theta+r_{L} u\left[T_{L} \cos \theta+(L-1) \sin \theta\right]\right\}, \\
\dot{\theta} & =r_{L}^{2}\left(\dot{y}_{L} x_{L}-y_{L} \dot{x}_{L}\right) \\
& =L \cos ^{2} \theta+\sin ^{2} \theta-T_{L} \cos \theta \sin \theta+r_{L} u\left[(L-1) \cos \theta-T_{L} \sin \theta\right] .
\end{aligned}
$$

We have the system

(23)

$$
r_{R}^{\prime}=\frac{\dot{r}_{R}}{\dot{\theta}}=\frac{-r_{R}\left\{T_{R} \cos ^{2} \theta+(R-1) \cos \theta \sin \theta-r_{R} v\left[T_{R} \cos \theta+(R-1) \sin \theta\right]\right\}}{R \cos ^{2} \theta+\sin ^{2} \theta-T_{R} \cos \theta \sin \theta-r_{R} v\left[(R-1) \cos \theta-T_{R} \sin \theta\right]}
$$

for $\theta \in[-\pi / 2, \pi / 2]$, and

$$
r_{L}^{\prime}=\frac{\dot{r}_{L}}{\dot{\theta}}=\frac{-r_{L}\left\{T_{L} \cos ^{2} \theta+(L-1) \cos \theta \sin \theta+r_{L} u\left[T_{L} \cos \theta+(L-1) \sin \theta\right]\right\}}{L \cos ^{2} \theta+\sin ^{2} \theta-T_{L} \cos \theta \sin \theta+r_{L} u\left[(L-1) \cos \theta-T_{L} \sin \theta\right]}
$$

for $\theta \in[\pi / 2,3 \pi / 2]$. In this case, the divergence of systems (23) and (24) on the periodic orbit (which is $r_{R}=0$ with $\theta \in[-\pi / 2, \pi / 2]$ in the right zone and $r_{L}=0$ with $\theta \in[\pi / 2,3 \pi / 2]$ in the left zone) is, using the divergence formula in polar coordinates, respectively

$$
\text { divergence }\left._{R}\right|_{r_{R}=0}=\left.\frac{1}{r_{R}} \frac{\partial\left(r_{R} r_{R}^{\prime}\right)}{\partial r_{R}}\right|_{r_{R}=0}=-2 \frac{\mathcal{P}\left(R, T_{R}\right)}{\mathcal{Q}\left(R, T_{R}\right)},
$$

and

where

$$
\text { divergence }\left._{L}\right|_{r_{L}=0}=\left.\frac{1}{r_{L}} \frac{\partial\left(r_{L} r_{L}^{\prime}\right)}{\partial r_{L}}\right|_{r_{L}=0}=-2 \frac{\mathcal{P}\left(L, T_{L}\right)}{\mathcal{Q}\left(L, T_{L}\right)},
$$

$$
\begin{aligned}
& \mathcal{P}(A, B)=B \cos ^{2} \theta+(A-1) \cos \theta \sin \theta, \\
& \mathcal{Q}(A, B)=1+(A-1) \cos ^{2} \theta-B \cos \theta \sin \theta .
\end{aligned}
$$


In view of Proposition 6 we must compute

$$
\mathcal{I}_{R L}=\int_{\pi / 2}^{3 \pi / 2} \text { divergence }\left._{L}\right|_{r_{L}=0} d \theta+\int_{-\pi / 2}^{\pi / 2} \text { divergence }\left._{R}\right|_{r_{R}=0} d \theta .
$$

For the calculations we can take advantage that

$$
\frac{d}{d \theta}\left[1+(A-1) \cos ^{2} \theta-B \cos \theta \sin \theta\right]=-2(A-1) \cos \theta \sin \theta-2 B \cos ^{2} \theta+B,
$$

so that, for instance, for the right part,

$$
-2 \int_{-\frac{\pi}{2}}^{\frac{\pi}{2}} \frac{B \cos ^{2} \theta+(A-1) \cos \theta \sin \theta}{1+(A-1) \cos ^{2} \theta-B \cos \theta \sin \theta} d \theta
$$

reduces to

$$
-\int_{-\frac{\pi}{2}}^{\frac{\pi}{2}} \frac{B}{1+(A-1) \cos ^{2} \theta-B \cos \theta \sin \theta} d \theta
$$

since

gives

$$
\int_{-\frac{\pi}{2}}^{\frac{\pi}{2}} \frac{-2\left[B \cos ^{2} \theta+(A-1) \cos \theta \sin \theta\right]+B}{1+(A-1) \cos ^{2} \theta-B \cos \theta \sin \theta} d \theta
$$

$$
\left[\log \left(1+(A-1) \cos ^{2} \theta-B \cos \theta \sin \theta\right)\right]_{-\frac{\pi}{2}}^{\frac{\pi}{2}}=0 .
$$

Similar reduction can be achieved for the left part. Doing these integrals, we get that

$$
\mathcal{I}_{R L}=-2 \pi\left(\frac{T_{R}}{\sqrt{4 R-T_{R}^{2}}}+\frac{T_{L}}{\sqrt{4 L-T_{L}^{2}}}\right) .
$$

By assumptions, see $(22), \mathcal{I}_{R L}>0$ and it follows from Proposition 6 that the periodic orbit at infinity is unstable.

Taking an orbit starting at a point $(0, M)$ with $M>0$ sufficiently big, the instability of the periodic orbit at infinity assures that after a turn the orbit will pass through a point $(0, m)$ with $0<m<M$, as in Figure 2 . Then, by joining these two points with a segment we have a positively invariant compact set $\Omega$ containing the period annulus. This concludes the proof of statement (FF) of Theorem 4.

Note that we could also study the (FF) case by computing the local expansion of Poincaré map at infinity, by resorting to Proposition 6 of [22] in order to cope with piecewise defined vector fields. If this alternative approach is used, then the hypotheses assure that the derivative of such Poincaré map at $r=0$ is greater than one and the same conclusion follows.

Lemma 11. The Liénard piecewise linear differential system (1) with $0<v<$ $u, T_{C}=0, T_{R}>0$ and $T_{L}<0$ cannot have an orbit $\gamma$ starting at $(x, y)=$ $\left(v, \sqrt{u^{2}-v^{2}}\right)$ and arriving after a turn at a point $(v, y)$ with $0<y<\sqrt{u^{2}-v^{2}}$, as in Figure 10.

Proof. If such an orbit exists, then we could close the orbit with a segment on the line $x=v$, building a positively invariant compact set $\Omega$. Thus, from Proposition 9 we should have a stable limit cycle $\gamma_{1}(t)$ contained in $\Omega$ and surrounding the period annulus. Now from Proposition 8 we know that a second unstable limit cycle $\gamma_{2}(t)$ appears for $T_{C}<0$ sufficiently small, and $\gamma_{1}(t)$ persists due to its stability. Note that $\gamma_{1}(t)$ and $\gamma_{2}(t)$ are contained in the half-space $x>-u$. But this is a 


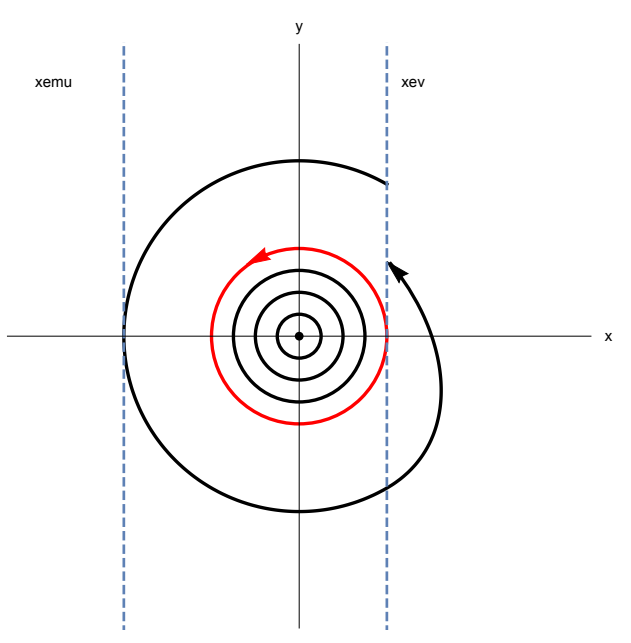

FIGURE 10. The orbit $\gamma$ starting at $(x, y)=\left(v, \sqrt{u^{2}-v^{2}}\right)$ and arriving after surrounding the period annulus at a point $(v, y)$ with $0<y<\sqrt{u^{2}-v^{2}}$, is not possible under hypotheses $0<v<u$, $T_{L}<0, T_{C}=0$, and $T_{R}>0$.

contradiction since a piecewise linear differential system with two zones separated by a straight line has at most one limit cycle (see $[8,21]$ ).

Proof of statement (NF) of Theorem 4. We recall that $0<v<u, T_{R}>0, T_{L}<0$ and

$$
L, R>0, T_{R}>2 \sqrt{R},\left|T_{L}\right|>2 \sqrt{L} .
$$

Note that under these assumptions system (1) has one virtual focus on the right hand zone, and a virtual node on the left hand zone. We shall describe the compact set $\Omega$ in the Poincaré disc, see Figure 3 .

The point $e_{L}$ is a virtual attractor node whose invariant straight lines $\Gamma_{ \pm}$with director vectors the eigenvectors of the linear part at $e_{L}$ intersect the line $x=-u$ at the points $\left(-u, P_{L}^{-}\right)$and $\left(-u, P_{L}^{+}\right)$with $P_{L}^{-}<P_{L}^{+}<0$, see Proposition 2(a.1) and Figure 3.

Since $P_{L}^{+}<0$ the orbit $\gamma(t)$ through $\left(-u, P_{L}^{+}\right)$crosses the band $-u<x<v$ and enters the right hand zone at the point $\left(v, Q_{L}^{+}\right)$, see (3). Since $\left.\dot{x}_{R}\right|_{x=v}=-y$ and $\left.\dot{y}_{R}\right|_{y=0}=R(x-v)+v>0$, the orbit $\gamma(t)$ exits the right hand zone. We note that $\gamma$ cannot intersect the segment $s_{1}$ on the line $x=v$ with $0 \leq y \leq \sqrt{u^{2}-v^{2}}$, otherwise we have an orbit satisfying the assumptions of Lemma 11, and this is not possible. So the orbit $\gamma(t)$ crosses again the central zone as in Figure 3, and since at infinite the straight line $\Gamma_{+}$has a saddle (see Figure 3 and use the Poincaré compactification of Appendix A), the orbit $\gamma(t)$ enters again the central zone. Using this orbit and an appropriate segment $s_{2}$ on the straight line $x=-u$, we obtain the compact set $\Omega$ homeomorphic to a closed disc surrounding the period annulus and positively invariant. This concludes the proof of statement (NF) of Theorem 4 . 
Proof of statement (NN) of Theorem 4. We recall that $0<v<u, T_{R}>0, T_{L}<0$ and

$$
L, R>0, T_{R}>2 \sqrt{R},\left|T_{L}\right|>2 \sqrt{L}, Q_{L}^{+}>P_{R}^{-} .
$$

Note that under these assumptions system (1) has two virtual nodes. We shall describe the compact set $\Omega$ in the Poincaré disc, see Figure 4. The point $e_{R}$ is a virtual unstable node whose invariant straight lines with director vectors the eigenvectors of the linear part of $e_{R}$ intersect the line $x=v$ on the points $\left(v, P_{R}^{-}\right)$ and $\left(v, P_{R}^{+}\right)$. From Proposition 3(a.1) we have $P_{R}^{+}<P_{R}^{-}<0$. Moreover the point $e_{L}$ is a virtual attractor node whose invariant straight lines with director vectors the eigenvectors of the linear part of $e_{L}$ intersect the line $x=-u$ on the points $\left(-u, P_{L}^{-}\right)$and $\left(-u, P_{L}^{+}\right)$. From Proposition 2(a.1) we have $P_{L}^{-}<P_{L}^{+}<0$. Now we compute the radius of the piece of circular orbit on the central zone passing through the point $\left(-u, P_{L}^{+}\right)$. Doing so we compute the point where this orbit intersects the line $x=v$ and we get the point $\left(v, Q_{L}^{+}\right)$, see (3). By assumptions we have that $Q_{L}^{+}>P_{R}^{-}$. Moreover using (31) and (32), at infinity we have two singular points at the endpoints of the invariant straight lines through $\left(-u, P_{L}^{+}\right)$and $\left(v, P_{R}^{-}\right)$being saddles. Hence we can compute the compact set $\Omega$ by following the orbit through the point $\left(v, Q_{L}^{+}\right)$, as in the previous case, or by using the segment joining the points $\left(v, P_{R}^{-}\right)$and $\left(v, Q_{L}^{+}\right)$, see Figure 4 . This completes the proof of statement $(\mathrm{NN})$ of Theorem 4.

Proof of statement (SF) of Theorem 4. We recall that $0<v<u, T_{R}>0, T_{L}<0$ and

$$
L<0, R>0, T_{R}<2 \sqrt{R}, H_{R}^{F}\left(v-\bar{x}_{R}, Q_{L}^{+}-\bar{y}_{R}\right)<H_{R}^{F}\left(v-\bar{x}_{R}, Q_{L}^{-}-\bar{y}_{R}\right) .
$$

Note that under these assumptions system (1) has one saddle on the left hand zone and a virtual focus on the right hand zone. We shall describe the compact set $\Omega$ in the Poincare disc, see Figure 5 . The point $e_{L}$ is a saddle whose invariant manifolds intersect the line $x=-u$ at the points $\left(-u, P_{L}^{-}\right)$and $\left(-u, P_{L}^{+}\right)$. By Proposition 2(a.2) we have $P_{L}^{+}<0<P_{L}^{-}$. Furthermore the point $e_{R}$ is a virtual unstable focus. Now we compute the radius of the circular orbit on the central zone passing through the point $\left(-u, P_{L}^{+}\right)$. Doing so we compute the point where this orbit intersects the line $x=v$ and we get the point $\left(v, Q_{L}^{+}\right)$, see (5). Now we compute the radius of circular orbit in the central zone passing through the point $\left(-u, P_{L}^{-}\right)$and then we compute the point where this orbit intersects the line $x=v$ in backward time and we get the point $\left(v, Q_{L}^{-}\right)$, see (5).

Recall that the focus dynamics in the right hand zone has the first integral $H_{R}^{F}(x, y)$ defined in (12). It is easy to conclude, by using the points on the vertical isocline $\tilde{y}=T_{R} \tilde{x}=2 \sigma_{R} \tilde{x}$ and that $H_{R}^{F}(x, y)$ increases with the $x$-value of the intersection point of the orbit with such isocline, that assumption (14) assures that the orbit through $\left(v, Q_{L}^{+}\right)$arrives at the line $x=v$ below the point $\left(v, Q_{L}^{-}\right)$. Then, we can obtain a positively invariant compact set $\Omega$ by joining this arrival point with the point $\left(v, Q_{L}^{-}\right)$, see Figure 5 . This completes the proof of statement (SF) of Theorem4.

Proof of statement (FS) of Theorem 4. We recall that $0<v<u, T_{R}>0, T_{L}<0$ and

$$
L>0, R<0,\left|T_{L}\right|<2 \sqrt{L}, H_{L}^{F}\left(-u-\bar{x}_{L}, Q_{R}^{+}-\bar{y}_{L}\right)<H_{L}^{F}\left(-u-\bar{x}_{L}, Q_{R}^{-}-\bar{y}_{L}\right) .
$$


Note that under these assumptions system (1) has one saddle on the right hand zone and a virtual focus on the left hand zone. We shall describe the compact set $\Omega$ in the Poincaré disc, see Figure 6 . The point $e_{R}$ is a saddle whose invariant manifolds intersect the line $x=v$ at the points $\left(v, P_{R}^{-}\right)$and $\left(v, P_{+, R}\right)$. By Proposition 3(a.2) we have $P_{R}^{-}<0<P_{R}^{+}$. Furthermore the point $e_{L}$ is a virtual unstable focus. Now we compute the radius of the circular orbit on the central zone passing through the point $\left(-u, P_{L}^{+}\right)$. Doing so we compute the point where this circular orbit intersects the line $x=v$ and we get the point $\left(v, Q_{L}^{+}\right)$. Now we compute the radius of the circular orbit in the central zone passing through the point $\left(-u, P_{L}^{-}\right)$and then we compute the point where this circular orbit intersects the line $x=v$ in backward time and we get the point $\left(v, Q_{L}^{-}\right)$.

The focus dynamics in the left hand zone has the first integral $H_{L}^{F}(x, y)$ defined in (7). By using the points on the vertical isocline $\tilde{y}=T_{L} \tilde{x}=2 \sigma_{L} \tilde{x}$ and that $H_{R}^{F}(x, y)$ increases with the absolute $|x|$-value of the intersection point of the orbit with such isocline, assumption (14) assures that the orbit through $\left(-u, Q_{R}^{+}\right)$arrives at the line $x=-u$ above the point $\left(-u, Q_{R}^{-}\right)$. Then, we can obtain a positively invariant compact set $\Omega$ by joining this arrival point with the point $\left(-u, Q_{R}^{-}\right)$, see Figure 6. This completes the proof of the statement (FS) of Theorem 4.

Proof of statement (SN) of Theorem 4. We recall that $0<v<u, T_{R}>0, T_{L}<0$ and

$L<0, R>0, T_{R}>2 \sqrt{R}, Q_{L}^{+}>P_{R}^{-}, H_{R}^{N}\left(v-\bar{x}_{R}, Q_{L}^{+}-\bar{y}_{R}\right)<H_{R}^{N}\left(v-\bar{x}_{R}, Q_{L}^{-}-\bar{y}_{R}\right)$.

Note that under these assumptions system (1) has one saddle on the left hand zone and a virtual node on the right hand zone. To describe the compact set $\Omega$ in the Poincaré disc, the arguments and the figure are essentially the same as in the statement (SF) and thus we do not provide them here, see Figure 7.

Proof of statement (NS) of Theorem 4. We recall that $0<v<u, T_{R}>0, T_{L}<0$ and

$$
L>0, R<0,\left|T_{L}\right|>2 \sqrt{L}, H_{L}^{N}\left(-u-\bar{x}_{L}, Q_{R}^{+}-\bar{y}_{L}\right)<H_{L}^{N}\left(-u-\bar{x}_{L}, Q_{R}^{-}-\bar{y}_{L}\right) .
$$

Note that under these assumptions system (1) has one saddle on the right hand zone and a virtual node on the left hand zone. To describe the compact set $\Omega$ in the Poincaré disc, the arguments and the figure are essentially the same as in the statement (FS) and thus we do not provide them, see Figure 8. Note however that if $P_{L}^{+}>Q_{R}^{-}$holds, then the orbit through $Q_{R}^{+}$arrives for sure to the line $x=-u$ above $Q_{R}^{-}$and so condition (16) is automatically fulfilled.

Proof of statement (SS) of Theorem 4. We recall that $0<v<u, T_{R}>0, T_{L}<0$ and

$$
L<0, R<0, Q_{L}^{+}>P_{R}^{-}, H_{R}^{S}\left(v-\bar{x}_{R}, Q_{L}^{+}-\bar{y}_{R}\right)>H_{R}^{S}\left(v-\bar{x}_{R}, Q_{L}^{-}-\bar{y}_{R}\right) .
$$

Note that here the saddle dynamics on the right zone has the first integral given in (11), which when evaluated at the points on the vertical isocline $\tilde{y}=T_{R} \tilde{x}=2 \sigma_{R} \tilde{x}$ becomes

$$
H_{R}^{S}\left(\tilde{x}, 2 \sigma_{R} \tilde{x}\right)=\log \sqrt{w_{R}^{2} \tilde{x}^{2}-\sigma_{R}^{2} \tilde{x}^{2}}+\frac{\sigma_{R}}{w_{R}} \operatorname{arctanh}\left(\frac{\sigma_{R}}{w_{R}}\right),
$$

and so it increases when $|\tilde{x}|=\left|x-\bar{x}_{R}\right|=\bar{x}_{R}-x$ increases. Then, the first integral decreases when the positive $x$-value of the intersection point of the orbit with such 
isocline increases, and to assure that the orbit through $Q_{L}^{+}$arrives at the line $x=v$ below $Q_{L}^{-}$we need a reversed inequality (compared with the SF and SN cases).

The proof follows exactly the same lines as the proofs of statements (SF) or (SN) and thus we do not repeat them. Note however that if $P_{R}^{+}<Q_{L}^{-}$holds, then the orbit through $Q_{L}^{+}$arrives for sure to the line $x=v$ below $Q_{L}^{-}$and so condition (18) is automatically fulfilled.

\section{Proof of Theorem 5}

To show Theorem 5, we first recall a necessary condition for existence of limit cycles which is obtained from applying the Filippov's transformations, see [32, 33]. Working in the set of $x$-values corresponding to $S_{\mathrm{RVF}}$, we define the function

$$
G(x)=\int_{0}^{x} g(s) d s
$$

positive for $x \neq 0$, since we exclude the possible regions with $x g(x)<0$. Thus, the function $G$ is only defined for $x \geq \bar{x}_{L}$ if $L<0$ and for $x \leq \bar{x}_{R}$ if $R<0$. Let $x_{L}(z)<0<x_{R}(z)$ be the two solution branches of the equation $G(x)=z>0$, where we also take $x_{L}(0)=x_{R}(0)=0$, and the domains of such functions could be different when real saddles appear. If we define the functions

$$
F_{\{L, R\}}(z)=F\left(x_{\{L, R\}}(z)\right),
$$

it turns out that the differential equation

$$
\frac{d y}{d z}=F_{R}(z)-y
$$

reproduces for $z \geq 0$ the orbits of system (1) for $x \geq 0$, while the differential equation

$$
\frac{d y}{d z}=F_{L}(z)-y
$$

reproduces also for $z \geq 0$ the orbits of system (1) for $x \leq 0$. This technique allows to 'fold' the phase plane and to apply comparison principles, so that for instance we can write the following result, which is a direct consequence of Theorem 5.4 of [32].

Proposition 12. For the differential systems (1) with $0<v<u, T_{R}>0, T_{L}<0$, $L \neq 0, R \neq 0$, a necessary condition for the existence of periodic orbits is the existence of a value $z^{*}>0$ such that $F_{L}\left(z^{*}\right)=F_{R}\left(z^{*}\right)$.

In what follows, we will see that, in all the cases of Theorem 4 , when $\left(-T_{C}\right)$ is big enough there are no intersections between the graphs of $F_{L}$ and $F_{R}$, so that Theorem 5 is a direct consequence of Proposition 12.

We start by computing

$$
G(x)= \begin{cases}\frac{1}{2}\left[L(x+u)^{2}-2 u(x+u)+u^{2}\right], & \text { if } x \leq-u, \\ \frac{1}{2} x^{2}, & \text { if }-u<x<v, \\ \frac{1}{2}\left[R(x-v)^{2}+2 v(x-v)+v^{2}\right], & \text { if } x \geq v,\end{cases}
$$


where we must add the restrictions $x+u>u / L$ or $x-v<-v / R$, whenever $L<0$ or $R<0$, respectively, or both if $L<0$ and $R<0$. Accordingly, we get

$$
x_{L}(z)= \begin{cases}-\sqrt{2 z}, & \text { if } 2 z \leq u^{2}, \\ -u-\frac{1}{L}\left(\sqrt{u^{2}+L\left(2 z-u^{2}\right)}-u\right), & \text { if } 2 z>u^{2},\end{cases}
$$

where we must add the restriction $2 z \leq u^{2}(1-1 / L)$ when $L<0$, and

$$
x_{R}(z)= \begin{cases}\sqrt{2 z}, & \text { if } 2 z \leq v^{2}, \\ v+\frac{1}{R}\left(\sqrt{v^{2}+R\left(2 z-v^{2}\right)}-v\right), & \text { if } 2 z>v^{2},\end{cases}
$$

where we must add the restriction $2 z \leq v^{2}(1-1 / R)$ when $R<0$. After composing these functions with the function $F$, we obtain

$$
F_{L}(z)= \begin{cases}-T_{C} \sqrt{2 z}, & \text { if } 2 z \leq u^{2} \\ -\frac{T_{L}}{L}\left(\sqrt{u^{2}+L\left(2 z-u^{2}\right)}-u\right)-T_{C} u, & \text { if } 2 z>u^{2}\end{cases}
$$

and

$$
F_{R}(z)= \begin{cases}T_{C} \sqrt{2 z}, & \text { if } 2 z \leq v^{2}, \\ \frac{T_{R}}{R}\left(\sqrt{v^{2}+R\left(2 z-v^{2}\right)}-v\right)+T_{C} v, & \text { if } 2 z>v^{2},\end{cases}
$$

where the graph of $F_{L}$ should be restricted to $2 z \leq u^{2}(1-1 / L)$ when $L<0$ and the graph of $F_{R}$ to $2 z \leq v^{2}(1-1 / R)$ when $R<0$.

Assume first that we are in one of the cases with $L>0$ and $R>0$, that is, we are in cases $(\mathrm{FF}),(\mathrm{NF})$ or $(\mathrm{NN})$, under the corresponding hypotheses of Theorem 4. We claim that by choosing $\left(-T_{C}\right)$ big enough we have $F_{L}(z)>F_{R}(z)$ for all $z>0$, so that from Proposition 12 we cannot have limit cycles and therefore the conclusion of this theorem holds.

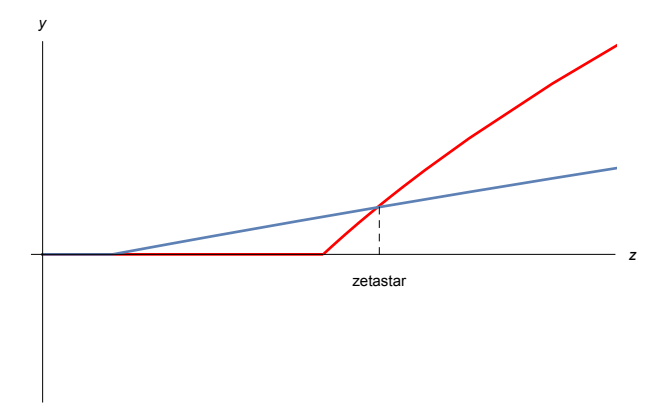

FiguRE 11. The graphs of the functions $F_{L}(z)$ (in red) and $F_{R}(z)$ (in blue) for $T_{C}=0$ under the hypotheses of $(\mathrm{FF})$ case. Note that $F_{L}(z)=0$ for $0<2 z<u^{2}$ and $F_{R}(z)=0$ for $0<2 z<v^{2}$.

To show the claim, note first that for $T_{C}=0$ the two graphs coincide for all $0 \leq 2 z \leq v^{2}$, since $v^{2}<u^{2}$ and then both functions vanish; this fact corresponds to the existence of the period annulus of statement (a) of Theorem 4. In any of these three cases of Theorem 4, we do know that there exists a stable limit cycle surrounding the period annulus, so that a straightforward extension of Proposition 
12 implies the existence of a value $z^{*}$ with $2 z^{*}>u^{2}$ where $F_{L}\left(z^{*}\right)=F_{R}\left(z^{*}\right)$. The situation is depicted in Figure 11 for the (FF) case of Table 1, where the fact that $F_{L}(z)<F_{R}(z)$ for $v^{2}<2 z<2 z^{*}$ tells us that orbits near the period annulus after a complete turn go far away from the annulus, agreeing with Proposition 7 . It is precisely the intersection at $z^{*}$ leading to $F_{L}(z)>F_{R}(z)$ for $z>z^{*}$ what allows the existence of a closed orbit (the stable limit cycle surrounding the period annulus) that does return at the same point of the initial condition taken on the negative part of $y$-axis. Furthermore, the hypotheses of cases (FF) and (NF) imply that the condition

$$
\frac{T_{L}}{\sqrt{L}}+\frac{T_{R}}{\sqrt{R}}<0
$$

is fulfilled. On the other hand, we have

$$
\left.\lim _{z \rightarrow \infty} \frac{F_{R}(z)}{F_{L}(z)}\right|_{T_{C}=0}=\lim _{z \rightarrow \infty} \frac{\frac{T_{R}}{R}\left(\sqrt{v^{2}+R\left(2 z-v^{2}\right)}-v\right)}{-\frac{T_{L}}{L}\left(\sqrt{u^{2}+L\left(2 z-u^{2}\right)}-u\right)}=\frac{T_{R} / \sqrt{R}}{-T_{L} / \sqrt{L}},
$$

so that condition (29) assures that the above limit is less than 1 and so $F_{L}(z)>$ $F_{R}(z)$ for all $z$ sufficiently big. Thus, since two parabolic branches have at most two intersections, we deduce that $F_{L}(z)>F_{R}(z)$ for all $z>z^{*}$ and therefore there is only one intersection in cases (FF) and (NF), as in Figure 11. This allows to define the positive quantity

$$
H=\max _{z \geq 0}\left[F_{R}(z)-F_{L}(z)\right]=\max _{0 \leq z \leq z^{*}}\left[F_{R}(z)-F_{L}(z)\right]>0 .
$$

If starting from the situation of Figure 11, we allow $T_{C}<0$ we see that for small values of $z$ the functions do not vanish any longer while the right parts of the graphs just undergo translations (recall (27)-(28)). Thus, for $2 z \geq u^{2}$ the graph of $F_{L}(z)$ goes up an amount equal to $\left(-T_{C} u\right)$, while for $2 z \geq v^{2}$ the graph of $F_{R}(z)$ goes down an amount equal to $T_{C} v$. Note that $F_{L}(z)>F_{R}(z)$ for all $0<2 z \leq v^{2}$, what indicates that the origin is now a stable focus, see the left panel of Figure 12. In the figure, we observe two transversal intersections between the two graphs that correspond for sure to the two limit cycles predicted in statement (b) of Theorem 4 when $T_{C}$ is small enough, but it should be remarked that we cannot identify in general the number of intersections with the number of limit cycles: the only valid assertion is, from Proposition 12, that without intersections there are no limit cycles. If we increase more the value of $\left(-T_{C}\right)$, namely by taking $\left(-T_{C}\right)(u+v)>H$, we get a situation without intersections between the graphs of $F_{L}(z)$ and $F_{R}(z)$, see the right panel of Figure 12. So, the above claim is proved and the theorem is completed for the cases $(\mathrm{FF})$ and $(\mathrm{NF})$.

In the (NN) case we cannot guarantee condition (29) so that there could have in principle two intersections when $T_{C}=0$, a situation leading to $F_{R}(z)-F_{L}(z)>0$ (and not bounded) for $z$ sufficiently big. Anyway, we do not have to consider all possibles values of $z>0$, since the stable limit cycle (and any other possible periodic orbit) must be surrounded by the orbit with initial condition $\left(v, Q_{L}^{+}\right)$, see Figure 4. Such an orbit defines a maximal value of $x_{M}$, so that the corresponding value $z_{M}$ with $x_{R}\left(z_{M}\right)=x_{M}$ bounds the interval where we must look for intersections of the graphs. Note that this bound is valid for all $T_{C}>0$ thanks to a property of rotated vector fields, which implies in our case that the stable limit cycle shrinks 
when $\left(-T_{C}\right)$ increases. Thus, if we now define the positive quantity

$$
H=\max _{0 \leq z \leq z_{M}}\left[F_{R}(z)-F_{L}(z)\right]>0,
$$

we can follow the same reasoning as before to conclude that for $\left(-T_{C}\right)$ big enough there are no intersections for $z<z_{M}$ and therefore no periodic orbits in the (NN) case.

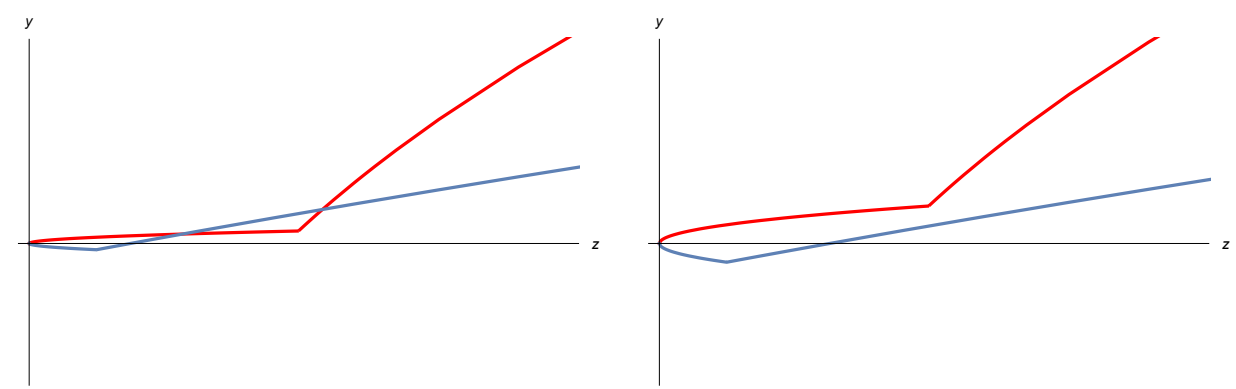

FiguRE 12. The graphs of the functions $F_{L}(z)$ (in red) and $F_{R}(z)$ (in blue) for $T_{C}<0$ and small in absolute value (left) and for $\left(-T_{C}\right)(u+v)>H$ (right) in the case $(\mathrm{FF})$ of Table 1 .

To show the cases where saddles zones are involved, we can follow a similar reasoning. The only difference is that at least one of the graphs is finite. Excepting in the (SS) case, we have now parabolic branches with different concavity. Again, as from Theorem 4 there is a stable limit cycle for $T_{C}=0$, we deduce the existence of at least one transversal intersection point $z^{*}$, and we can define the maximum value $H$ for the difference $F_{R}(z)-F_{L}(z)$ (this time on the common maximal definition interval for the two functions) and assure the existence of a value of $T_{C}<0$ from which there are no intersections. We illustrate these cases by showing some instances of them in Figure 13. The proof is completed.
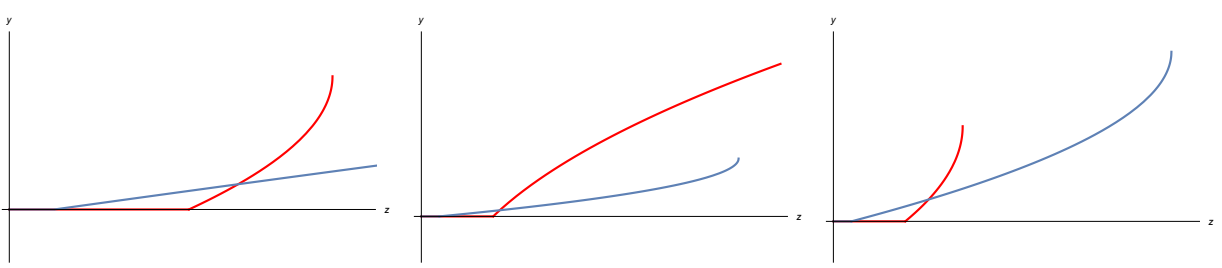

Figure 13. The graphs of the functions $F_{L}(z)$ (in red) and $F_{R}(z)$

(in blue) for the cases (SN), (FS) and (SS) of Table 1 when $T_{C}=0$.

Before ending the work, we write a couple of remarks.

Remark 13. For all the cases of Theorem 4, we can obtain a condition on $T_{C}<0$ that implies no intersections for the graphs of $F_{R}(z)$ and $F_{R}(z)$ when $0<2 z<u^{2}$. To do this, it suffices to consider the range $v^{2}<2 z<u^{2}$, since for $T_{C}<0$ we know that $F_{L}(z)>F_{R}(z)$ when $0<2 z \leq v^{2}$. Thus, we impose that

$$
-T_{C} \sqrt{2 z}>\frac{T_{R}}{R}\left(\sqrt{v^{2}+R\left(2 z-v^{2}\right)}-v\right)+T_{C} v
$$


which is equivalent to

$$
-T_{C}(\sqrt{2 z}+v)>\frac{T_{R}}{R}\left(\sqrt{v^{2}+R\left(2 z-v^{2}\right)}-v\right)=\frac{T_{R}\left(2 z-v^{2}\right)}{\sqrt{v^{2}+R\left(2 z-v^{2}\right)}+v} .
$$

Since

it suffices to get

$$
\frac{T_{R}}{\sqrt{R}} \sqrt{2 z-v^{2}}=\frac{T_{R}\left(2 z-v^{2}\right)}{\sqrt{R\left(2 z-v^{2}\right)}}>\frac{T_{R}\left(2 z-v^{2}\right)}{\sqrt{v^{2}+R\left(2 z-v^{2}\right)}+v},
$$

$$
-T_{C}(\sqrt{2 z}+v)>\frac{T_{R}}{\sqrt{R}} \sqrt{2 z-v^{2}},
$$

which is true for $-T_{C}>T_{R} / \sqrt{R}$. In short, the condition

$$
T_{C}+\frac{T_{R}}{\sqrt{R}}<0
$$

assures that there is no limit cycle totally contained in the half-plane $x \geq-u$. So, the unstable limit cycle that bifurcates at $T_{C}=0$, if it still exists, lives in the three zones.

Remark 14. As mentioned before, Theorem 4 does not includes the (FN) case. The reasons that make this case different can now be clarified. Under the corresponding hypotheses $0<v<u, T_{R}>0, T_{C}=0, T_{L}<0, L, R>0,\left|T_{L}\right|<2 / \sqrt{L}$ and $T_{R}>2 / \sqrt{R}$, condition (29) is violated, since

$$
\frac{T_{L}}{\sqrt{L}}+\frac{T_{R}}{\sqrt{R}}>-2+2=0
$$

This together with (30) implies that $F_{R}(z)>F_{L}(z)$ not only for all $z$ with $v^{2}<$ $2 z \leq u^{2}$ (where $F_{L}(z)=0$ ) but also for $z$ sufficiently big (and possibly for all $z$ with $\left.2 z>v^{2}\right)$. In this case, if we allow $T_{C}<0$ then we have always at least one intersection for the graphs of $F_{R}(z)$ and $F_{L}(z)$. In fact, by integrating backwards in time the upper invariant manifold of the virtual node, it can be easily shown the existence of one unstable limit cycle for all values of $T_{C}<0$.

\section{Appendix A: Poincaré Compactification}

In this section we summarize the Poincaré compactification that we shall use for describing the global phase portraits of our systems. For more details on the Poincaré compactification see chapter 5 of [5].

Let $\mathbb{S}^{2}$ be the set of points $\left\{\left(s_{1}, s_{2}, s_{3}\right) \in \mathbb{R}^{3}: s_{1}^{2}+s_{2}^{2}+s_{3}^{2}=1\right\}$. We will call this sphere the Poincaré sphere. Given a polynomial vector field

$$
X=\left(\dot{x}_{1}, \dot{x}_{2}\right)=\left(P\left(x_{1}, x_{2}\right), Q\left(x_{1}, x_{2}\right)\right)
$$

in $\mathbb{R}^{2}$, it can be extended analytically to the Poincaré sphere by projecting each point $\left(x_{1}, x_{2}\right) \in \mathbb{R}^{2}$ identified with the point $\left(x_{1}, x_{2}, 1\right) \in \mathbb{R}^{3}$ onto the Poincaré sphere using the straight line through $\left(x_{1}, x_{2}, 1\right)$ and the origin of $\mathbb{R}^{3}$. In this way we obtain two copies of $X$ : one on the northern hemisphere $\left\{\left(s_{1}, s_{2}, s_{3}\right) \in \mathbb{S}^{2}: s_{3}>0\right\}$, and another on the southern hemisphere $\left\{\left(s_{1}, s_{2}, s_{3}\right) \in \mathbb{S}^{2}: s_{3}<0\right\}$. The equator $\mathbb{S}^{1}=\left\{\left(s_{1}, s_{2}, s_{3}\right) \in \mathbb{S}^{2}: s_{3}=0\right\}$ corresponds to the infinity of $\mathbb{R}^{2}$. The local charts that we use for doing the calculations on the Poincaré sphere are

$$
U_{i}=\left\{\left(s_{1}, s_{2}, s_{3}\right) \in \mathbb{S}^{2}: s_{i}>0\right\}, \quad V_{i}=\left\{\left(s_{1}, s_{2}, s_{3}\right) \in \mathbb{S}^{2}: s_{i}<0\right\},
$$


with the corresponding local maps

$$
\phi_{i}\left(s_{1}, s_{2}, s_{3}\right): U_{i} \rightarrow \mathbb{R}^{2}, \quad \psi_{i}\left(s_{1}, s_{2}, s_{3}\right): V_{i} \rightarrow \mathbb{R}^{2},
$$

such that $\phi_{i}\left(s_{1}, s_{2}, s_{3}\right)=-\psi_{i}\left(s_{1}, s_{2}, s_{3}\right)=\left(\frac{s_{m}}{s_{i}}, \frac{s_{n}}{s_{i}}\right)$ for $m<n$ and $m, n \neq i$, for $i=1,2,3$. The expression for the corresponding vector field on $\mathbb{S}^{2}$ in the local chart $U_{1}$ is given by

$$
\dot{u}=v^{d}\left[-u P\left(\frac{1}{v}, \frac{u}{v}\right)+Q\left(\frac{1}{v}, \frac{u}{v}\right)\right], \quad \dot{v}=-v^{d+1} P\left(\frac{1}{v}, \frac{u}{v}\right) ;
$$

the expression for $U_{2}$ is

$$
\dot{u}=v^{d}\left[P\left(\frac{u}{v}, \frac{1}{v}\right)-u Q\left(\frac{u}{v}, \frac{1}{v}\right)\right], \quad \dot{v}=-v^{d+1} Q\left(\frac{u}{v}, \frac{1}{v}\right)
$$

and the expression for $U_{3}$ is just

$$
\dot{u}=P(u, v), \quad \dot{v}=Q(u, v),
$$

where $d$ is the degree of the vector field $X$. The expressions for the charts $V_{i}$ are the same than for the charts $U_{i}$ multiplied by $(-1)^{d-1}$, for $i=1,2,3$. Hence, to study the vector field $X$, it is enough to study its Poincaré compactification restricted to the northern hemisphere plus $\mathbb{S}^{1}$, which we will call the Poincaré disc and we will denote it by $\mathbb{D}$. To draw the phase portraits, we will consider the so-called gnomonic projection of the Poincaré disc on $\mathbb{R}^{2}$ by

$$
\pi\left(s_{1}, s_{2}, s_{3}\right)=\frac{1}{s_{3}+1}\left(s_{1}, s_{2}\right)=\frac{1}{1+\sqrt{1+x_{1}^{2}+x_{2}^{2}}}\left(x_{1}, x_{2}\right)
$$

where

$$
\left(s_{1}, s_{2}, s_{3}\right)=\frac{1}{\sqrt{1+x_{1}^{2}+x_{2}^{2}}}\left(x_{1}, x_{2}, 1\right)
$$

see Section 2.9.1 of [24] for more details.

Finite singular points of $X$ are the singular points of its compactification which are in $\mathbb{S}^{2} \backslash \mathbb{S}^{1}$, and they can be studied using $U_{3}$. Infinite singular points of $X$ are the singular points of the corresponding vector field on the Poincaré disc lying on $\mathbb{S}^{1}$. Since $\left(s_{1}, s_{2}, 0\right) \in \mathbb{S}^{1}$ is an infinite singular point if and only if $-\left(s_{1}, s_{2}, 0\right) \in \mathbb{S}^{1}$ is also an infinite singular point, and the local phase portrait of one is that of the other multiplied by $(-1)^{d-1}$, to study the infinite singular points it suffices only to look at $\left.U_{1}\right|_{v=0}$ and at the origin of $U_{2}$.

The next theorem (see [27]) gives a characterization of two topologically equivalent vector fields in the Poincaré disc. We recall that the separatrix skeleton of a differential system on the Poincaré disc $\mathbb{D}$ consists of all the separatrices of this differential system. See section 1.9 of [5] for the definition of separatrix. We also say that two differential systems on the Poincaré disc $\mathbb{D}$ are topologically equivalent if there exists a homeomorphism from $\mathbb{D}$ to $\mathbb{D}$ that maps the orbits of one system to the orbits of the other system preserving the orientation.

Theorem 15 (Markus-Neumann-Peixoto Theorem). Assume that $\left(\mathbb{D}, \phi_{1}\right)$ and $\left(\mathbb{D}, \phi_{2}\right)$ are two continuous flows with only isolated singular points. Then these flows are topologically equivalent if and only if their completed separatrix skeletons are topologically equivalent. 
This theorem implies that once the separatrices of a vector field in the Poincaré disc are determined, the global phase portrait of that vector field is obtained up to topological equivalence.

\section{ACKNOWLEDGEMENTS}

The first author is partially supported by a MINECO/FEDER grant MTM200803437, a CIRIT grant number 2014SGR-568, an ICREA Academia, two grants FP7-PEOPLE-2012-IRSES 316338 and 318999, and FEDER-UNAB10-4E-378.

The second author was supported by MINECO/FEDER grant MTM2015-65608$\mathrm{P}$ and by the Consejería de Economía y Conocimiento de la Junta de Andalucía under grant P12-FQM-1658.

The third author was partially supported by Portuguese National Funds through FCT - Fundação para a Ciência e a Tecnologia within the project UID/MAT/04459/2013.

\section{REFERENCES}

[1] T. Carletti and G. Villari, A note on existence and uniqueness of limit cycles for Liénard systems, J. Math. Anal. Appl. 307 (2005), 763-773.

[2] V. Carmona, E. Freire, E. Ponce and F. Torres, On simplifying and classifying piecewise linear systems, IEEE Trans. Circuits and Systems I: Fundamental Theory and Applications 49 (2002), 609-620.

[3] H. Chen, D. LI, J. XIE AND Y. Yue, Limit cycles in planar continuous piecewise linear systems, Commun. Nonlinear Sci. Numer. Simulat. 47 (2017), 438-454.

[4] F. Dumortier AND C. LI, On the uniqueness of limit cycles surrounding one or more singularities for Liénard equations, Nonlinearity 9 (1996), 1489-1500.

[5] F. Dumortier, J. Llibre And J.C. Artés, Qualitative theory of planar differential systems, Universitext, Springer-Verlag, Berlin, 2006.

[6] F. Dumortier And C. Rousseau, Cubic Liénard equations with linear damping, Nonlinearity 3 (1990), 1015-1039.

[7] E. Freire, E. PONCE AND J. Ros, Limit cycle bifurcation from center in symmetric piecewise-linear systems, Int. J. Bifurcation and Chaos 9 (1999), 895-907.

[8] E. Freire, E. Ponce, F. Rodrigo And F. Torres, Bifurcation sets of continuous piecewise linear systems with two zones, Int. J. Bifurcations and Chaos 8 (1998), 2073-2097.

[9] E. Freire, E. Ponce, F. Rodrigo And F. Torres, Bifurcation sets of continuous piecewise linear systems with three zones, Int. J. Bifurcations and Chaos 12 (2002), 1675-1702.

[10] E. Freire, E. Ponce And F. TORRes, Hopf-like bifurcations in planar piecewise linear systems, Publicacions Matemàtiques 41 (1997), 135-148.

[11] E. Freire, E. Ponce And F. Torres, A general mechanism to generate three limit cycles in planar Filippov systems with two zones, Nonlinear Dyn. (2014), DOI 10.1007/s11071-0141437-7.

[12] A. Gasull, H. Giacomini and J. Llibre, New criteria for the existence and non-existence of limit cycles in Liénard differential systems, Dynamical Systems: An International Journal 24 (2009), 171-185.

[13] D. Hilbert, Mathematische Probleme, Lecture, Second Internat. Congr. Math. (Paris, 1900), Nachr. Ges. Wiss. G"ttingen Math. Phys. KL. (1900), 253-297; English transl., Bull. Amer. Math. Soc. 8 (1902), 437-479.

[14] S. J. Hogan, Relaxation oscillations in a system with a piecewise smooth drag coefficiente, J. Sound and Vibration 263 (2003), 467-471.

[15] W. T. VAN Horssen, On oscillations in a system with a piecewise smooth coefficient, J. Sound and Vibration 283 (2005), 1229-1234.

[16] Yu. Ilyashenko, Centennial history of Hilbert's 16th problem, Bull. Amer. Math. Soc. 39 (2002), 301-354.

[17] A.I. Khibnik, B. Krauskopf and C. Rousseau, Global study of a family of cubic Liénard equations, Nonlinearity 11 (1998), 1505-1519.

[18] J. Li, Hilbert's 16th problem and bifurcations of planar polynomial vector fields, Internat. J. Bifur. Chaos Appl. Sci. Engrg. 13 (2003), 47-106. 
[19] M. Lima, C. Pessoa And W. F. Pereira, Limit cycles bifurcating from a period annulus in continuous piecewise linear differential systems with three zones, Internat. J. Bifur. Chaos Appl. Sci. Engrg. 27 (2017), 1750022 (14 pages).

[20] J. Llibre, A.C. Mereu AND M.A. Teixeira, Limit cycles of the generalized polynomial Liénard differential equations, Math. Proceed. Camb. Phyl. Soc. 148 (2009), 363-383.

[21] J. Llibre, M. Ordóñez AND E. Ponce, On the existence and uniqueness of limit cycles in a planar piecewise linear systems without symmetry, Nonlinear Analysis Series B: Real World Applications 14 (2013), 2002-2012.

[22] J. Llibre AND E. Ponce, Bifurcation of a periodic orbit from infinity in planar piecewise linear vector fields, Nonlinear Analysis 36 (1999), 623-653.

[23] J. Llibre And J. Sotomayor, Phase portraits of planar control systems, Nonlinear Analysis, Methods \& Applications 27 (1996), 1177-1197.

[24] J. Llibre AND A. Teruel, Introduction to the qualitative theory of differential systems, Birkhäuser, Advanced Texts, 2014.

[25] J. LlibRe AND C. VALLs, Limit cycles for a generalization of Liénard polynomial differential systems, Chaos, Solitons and Fractals 46 (2013), 65-74.

[26] J. Llibre, E. PONCE AND C. VAlls, Uniqueness and non-uniqueness of limit cycles of piecewise linear differential systems with three zones and no symmetry, J. Nonlin. Sci. 25 (2015), $861-867$.

[27] D. Neumann, Classification of continuous flows on 2-manifolds, Proc. Amer. Math. Soc. 48 (1975), 73-81.

[28] E. Ponce, J. Ros And E. Vela, Limit cycle and boundary equilibrium bifurcations in continuous planar piecewise linear systems, To appear in Internat. J. Bifur. Chaos Appl. Sci. Engrg. (2015).

[29] E. Ponce, J. Ros And E. VEla, The focus-center-limit cycle bifurcation in discontinuous planar piecewise linear systems without sliding, in Progress and Challenges in Dynamical Systems (S. Ibáñez, J. S. Pérez del Río, A. Pumari?o and J.A. Rodríguez, editors), Springer Proceedings in Mathematics \& Statistics 54 (2013) 335-349.

[30] S. Smale, Mathematical Problems for the Next Century, Mathematical Intelligencer 20 (1998), 7-15.

[31] D. Xiao And Z. Zhang, On the uniqueness and nonexistence of limit cycles for predator-prey systems, Nonlinearity 16 (2003), 1185-1201.

[32] Ye Yan-Qian and Others, Theory of Limit Cycles, Translations of Math. Monographs, Vol. 66, Amer. Math. Soc, Providence, 1986.

[33] Z. Zhang, T. Ding, W. HuAng, And Z. Dong, Qualitative Theory of Differential Equations, Translations of Math. Monographs, Vol. 101, Amer. Math. Soc, Providence, 1992.

1 Departament de Matemàtiques, Universitat Autònoma de Barcelona, 08193 Bellaterra, Barcelona, Catalonia, Spain E-mail address: jllibre@mat.uab.cat

2 Departamento de Matemática Aplicada, Escuela Técnica Superior de Ingeniería, Avda. De los Descubrimientos, 41092 Sevilla, Spain

E-mail address: eponcem@us.es

3 Departamento de Matemática, Instituto Superior Técnico, Universidade de Lisboa, 1049-001 Lisboa, Portugal

E-mail address: cvalls@math.tecnico.ulisboa.pt 